\title{
Sulphur-bearing species in the star forming region L1689N
}

\author{
V. Wakelam ${ }^{1}$, A. Castets ${ }^{1}$, C. Ceccarelli ${ }^{1,2}$, B. Lefloch ${ }^{2}$, E. Caux ${ }^{3}$, and L. Pagani ${ }^{4}$ \\ 1 Observatoire de Bordeaux, BP 89, 33270 Floirac, France \\ ${ }^{2}$ Laboratoire d'Astrophysique, Observatoire de Grenoble, BP 53, 38041 Grenoble Cedex 9, France \\ 3 CESR CNRS-UPS, BP 4346, 31028, Toulouse Cedex 04, France \\ ${ }^{4}$ LERMA \& FRE 2460 du CNRS, Observatoire de Paris, 61 Av. de l’Observatoire, 75014 Paris, France
}

Received 10 June 2002 / Accepted 7 October 2003

\begin{abstract}
We report observations of the expected main S-bearing species ( $\mathrm{SO}, \mathrm{SO}_{2}$ and $\mathrm{H}_{2} \mathrm{~S}$ ) in the low-mass star forming region L1689N. We obtained large scale $\left(\sim 300^{\prime \prime} \times 200^{\prime \prime}\right)$ maps of several transitions from these molecules with the goal to study the sulphur chemistry, i.e. how the relative abundances change in the different physical conditions found in L1689N. We identified eight interesting regions, where we carried out a quantitative comparative study: the molecular cloud (as reference position), five shocked regions caused by the interaction of the molecular outflows with the cloud, and the two protostars IRAS 16293-2422 and 16293E. In the cloud we carefully computed the gas temperature and density by means of a non-LTE LVG code, while in other regions we used previous results. We hence derived the column density of $\mathrm{SO}, \mathrm{SO}_{2}$ and $\mathrm{H}_{2} \mathrm{~S}$, together with $\mathrm{SiO}$ and $\mathrm{H}_{2} \mathrm{CO}$ - which were observed previously - and their relevant abundance ratios. We find that $\mathrm{SiO}$ is the molecule that shows the largest abundance variations in the shocked regions, whereas S-bearing molecules show more moderate variations. Remarkably, the region of the brightest $\mathrm{SiO}$ emission in $\mathrm{L} 1689 \mathrm{~N}$ is undetected in $\mathrm{SO}_{2}, \mathrm{H}_{2} \mathrm{~S}$ and $\mathrm{H}_{2} \mathrm{CO}$ and only marginally detected in $\mathrm{SO}$. In the other weaker $\mathrm{SiO}$ shocks, $\mathrm{SO}_{2}$ is enhanced with respect to $\mathrm{SO}$. We propose a schema in which the different molecular ratios correspond to different ages of the shocks. Finally, we find that $\mathrm{SO}, \mathrm{SO}_{2}$ and $\mathrm{H}_{2} \mathrm{~S}$ have significant abundance jumps in the inner hot core of IRAS 16293-2422 and discuss the implications of the measured abundances.
\end{abstract}

Key words. ISM: abundances - ISM: molecules - stars: formation - ISM: individual objects: L1689N -

ISM: individual objects: IRAS 16293-2422

\section{Introduction}

Low mass star forming regions are composed by at least three main ingredients: the molecular cloud from which protostars are born, the protostars themselves, and the shocked regions at the interface between the cloud and the outflows emanating from the protostars. These three regions have very different physical conditions, where temperature, density and also chemical abundances greatly differ (e.g. van Dishoeck \& Blake 1998). This paper focuses on the abundance changes occurring to the S-bearing molecules and the relevant sulphur chemistry. Depending on the physical condition of the gas, it is believed that different types of reactions play a role in the formation of sulphur-bearing molecules. In molecular clouds, ion-molecule reactions are the most important (Oppenheimer \& Dalgarno 1974; Prasad \& Huntress 1982; Millar \& Herbst 1990), whereas in the warm gas of the hot cores and shocks, neutral-neutral reactions play the major role in forming sulphur species (Pineau Des Forêts et al. 1993; Charnley 1997; Hatchell et al. 1998; Keane et al. 2001). Specifically, in warm

Send offprint requests to: V. Wakelam,

e-mail: wakelam@observ.u-bordeaux.fr gas, the abundances of $\mathrm{H}_{2} \mathrm{~S}, \mathrm{SO}$ and $\mathrm{SO}_{2}$ are supposed to increase significantly. This is the reason why they are often used to trace shocks (Pineau Des Forêts et al. 1993; Chernin et al. 1994; Bachiller \& Perez Gutierrez 1997). And because of the relatively fast evolution of their chemistry, on time scale of tens of thousand years, they are good candidates to be chemical clocks to study the evolution of outflows (Bachiller et al. 2001) and hot cores (Charnley 1997; Hatchell et al. 1998). Overall, it is widely accepted that in star forming regions the formation of S-bearing molecules is largely determined during the cold collapse phase, when atomic sulphur freezes out on grains and probably forms $\mathrm{H}_{2} \mathrm{~S}$. When the protostar starts to heat its environment, $\mathrm{H}_{2} \mathrm{~S}$ evaporates and it reacts with hydrogen atoms to give sulphur atoms. S rapidly reacts with $\mathrm{OH}$ and $\mathrm{O}_{2}$ to form $\mathrm{SO}$, that in turn gives $\mathrm{SO}_{2}$ by reacting with $\mathrm{OH}$ (e.g. Charnley 1997).

In this paper, we present large scale maps of several transitions of $\mathrm{SO}, \mathrm{SO}_{2}$ and $\mathrm{H}_{2} \mathrm{~S}$ in the molecular cloud L1689N, a molecular cloud located in the $\rho$ Ophiuchi cloud complex at $120 \mathrm{pc}$ from the Sun (Knude \& Hog 1998). Based on atomic oxygen observations, Caux et al. (1999) found that the gas temperature in this cloud is $(26 \pm 0.5) \mathrm{K}$ and the $\mathrm{H}_{2}$ density 
is larger than $3 \times 10^{4} \mathrm{~cm}^{-3}$. L1689N harbors two young protostellar sources. The first one is IRAS 16293-2422 (hereinafter IRAS 16293), a Class 0 protostar $\left(15 L_{\odot}\right)$ still in the accretion phase (Walker et al. 1986; Zhou 1995; Narayanan et al. 1998; Ceccarelli et al. 2000a). Like many other young protostars, IRAS 16293 is a binary system with a total mass around $1.1 M_{\odot}$ (Looney et al. 2000), whose two sources are separated by $5^{\prime \prime}$, namely a projected separation of $600 \mathrm{AU}$. The structure of the envelope surrounding IRAS 16293 has been reconstructed based on multifrequency $\mathrm{H}_{2} \mathrm{O}, \mathrm{SiO}, \mathrm{O}$ and $\mathrm{H}_{2} \mathrm{CO}$ line observations (Ceccarelli et al. 2000a,b). In the outer region $(r \geq 150 \mathrm{AU})$, the envelope gas shows molecular abundances typical of cold molecular clouds. In the inner region ( $r \leq 150 \mathrm{AU}$, i.e. about $2^{\prime \prime}$ in diameter) the abundances of $\mathrm{H}_{2} \mathrm{O}, \mathrm{SiO}$ and $\mathrm{H}_{2} \mathrm{CO}$ jump to abundances typical of the hot cores around massive protostars. This structure has been recently confirmed by Schöier et al. (2002), who modeled the continuum and the line emission from several other molecules. The second protostar, 16293E, is a recently discovered low mass and very young Class 0 source situated SouthEast of IRAS 16293. It was detected first by Mizuno et al. (1990) as a strong $\mathrm{NH}_{3}$ peak emission. Its protostellar nature is discussed in Castets et al. (2001, hereinafter CCLCL01).

The L1689N region is complex and has long been known to house multiple outflows (Fukui et al. 1986; Wootten \& Loren 1987; Mizuno et al. 1990; Hirano et al. 2001). The recent work by CCLCL01 claims that the two protostars IRAS 16293 and $16293 \mathrm{E}$ drive three bipolar outflows. Two of them originate from each of the two components of IRAS 16293, while the third outflow probably emanates from 16293E (Fig. 1). In the present article we will adopt the scheme outlined in CCLCL01 (Fig. 1), but our main conclusions are substantially unaffected by the actual evolutionary stage (pre-stellar or protostellar) of 16293E, questioned in Lis et al. (2002). What is important in the following discussion is the presence in $\mathrm{L} 1689 \mathrm{~N}$ of at least one protostar, IRAS 16293, and six regions which shows an enhancement of $\mathrm{SiO}$ and/or $\mathrm{H}_{2} \mathrm{CO}$ emission, and that are sites of shocked gas marked as E1, E2, HE1 ${ }^{1}, \mathrm{HE} 2, \mathrm{~W} 1$ and W2 (Fig. 1). In particular, CCLCL01 found that the brightest site of $\mathrm{SiO}$ emission, E2, does not show up any $\mathrm{H}_{2} \mathrm{CO}$ enhanced emission, whereas the brightest $\mathrm{H}_{2} \mathrm{CO}$ emission site, $\mathrm{E} 1$, is also accompanied by strong $\mathrm{SiO}$ emission. HE2 and HE1 represent a third class, for only $\mathrm{H}_{2} \mathrm{CO}$ emission is detected there and no $\mathrm{SiO}$. The goal of the present work is to study how the abundances of S-bearing molecules change in all these sites, compared with the abundances in the IRAS 16293 protostar and in the cloud.

The article is organized as follows. The observations are presented in Sect. 2, the results are presented in Sect. 3, and the column density determinations are detailed in Sect. 4. In Sect. 5 we discuss the results, i.e. the observed changes of $\mathrm{SO}, \mathrm{SO}_{2}$ and $\mathrm{H}_{2} \mathrm{~S}$ abundances with respect to the previously measured $\mathrm{SiO}$ and $\mathrm{H}_{2} \mathrm{CO}$ abundances and what this may teach us.

\footnotetext{
${ }^{1}$ This region is not considered farther in this work because too weak.
}

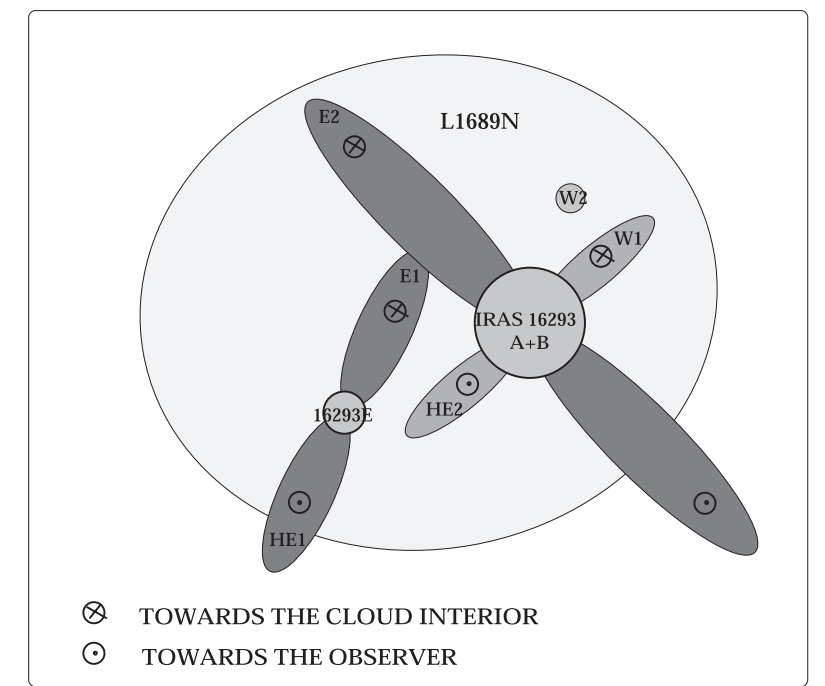

Fig. 1. Sketch of the region seen face on. The dark grey outflows have been clearly identified by CCLCL01. Light gray used for the outflow emanating from IRAS 16293 and the W2 source indicates that these are only assumptions.

\section{Observations}

Observations of large scale maps and specific positions inside the L1689N region were performed with the IRAM and SEST telescopes. We measured the emission of the following molecules: $\mathrm{SO}\left(3_{2} \rightarrow 2_{1}\right.$ and $6_{5} \rightarrow 5_{4}$ transitions), $\mathrm{SO}_{2}\left(5_{1,5} \rightarrow\right.$ $4_{0,4}$ transition $)$ and $\mathrm{H}_{2} \mathrm{~S}\left(2_{2,0} \rightarrow 2_{1,1}\right.$ and $1_{1,0} \rightarrow 1_{0,1}$ transitions). We also observed the ${ }^{34} \mathrm{SO} 3_{2} \rightarrow 2_{1}$ line in a few positions in order to estimate the opacity of the main isotopic line. The coordinates $(\Delta \alpha, \Delta \delta)$ of all maps shown here are offsets relative to the position of the 16293B component of the binary system IRAS 16293 at $\alpha(2000.0)=16^{\mathrm{h}} 32^{\mathrm{m}} 22^{\mathrm{s}} \cdot 6$, $\delta(2000.0)=-24^{0} 28^{\prime} 33^{\prime \prime}$ (the 16293A component is located $4^{\prime \prime}$ South and 2" East from the B component - Looney et al. 2000).

In June 1997 we obtained a map covering 120" $\times 100^{\prime \prime}$ in the $\mathrm{SO}_{2}$ molecular line with the IRAM-30m telescope. In November 2001, May and September 2002 we performed additional IRAM observations of the $\mathrm{SO}_{2},{ }^{34} \mathrm{SO}$ and $\mathrm{H}_{2} \mathrm{~S} 1_{1,0} \rightarrow$ $1_{0,1}$ lines at some specific positions in $\mathrm{L} 1689 \mathrm{~N}$, namely the shocked regions E1, E2, W1, W2 and HE2 (see Fig. 1 and the Introduction), the two protostars IRAS 16293 and $16293 \mathrm{E}$, and the molecular cloud (at the position $\Delta \alpha=120^{\prime \prime}, \Delta \delta=0^{\prime \prime}$ ). In the following we will refer to these eight positions as the "key" positions. The SEST telescope was used in July 1998 to map an area covering $300^{\prime \prime} \times 200^{\prime \prime}$ in the SO molecular lines and to map a smaller area covering $120^{\prime \prime} \times 100^{\prime \prime}$ in the $\mathrm{H}_{2} \mathrm{~S}$ molecular transition. Table 1 summarizes the different observed molecular transitions, together with their frequencies and upper level energies.

All data were obtained in the position switching mode with an OFF position located at $\Delta \alpha=-180^{\prime \prime}, \Delta \delta=0^{\prime \prime}$ from the center of IRAS 16293. Using the frequency switching mode we checked that this position is free of any $\mathrm{C}^{18} \mathrm{O}$ and ${ }^{13} \mathrm{CO}$ emission. Even though our observations are not Nyquist sampled, we smoothed our data to the largest beamsize when necessary, which is accurate enough, except in the case of pathological 
Table 1. Observed molecules together with the observing parameters. The critical densities $\left(n_{\text {cr }}\right)$ are computed for temperatures between 50 and $100 \mathrm{~K}$ using the collisional coefficients quoted in Sect. 4. The area mapped $(\Delta \alpha \times \Delta \delta)$ in each transition and the sampling of the map are reported in the last two lines. $B_{\text {eff }}$ is the beam efficiency of the telescope. The symbol "-" implies that only observations towards the "key" positions have been obtained.

\begin{tabular}{|c|c|c|c|c|c|c|c|c|}
\hline Transitions & $\mathrm{SO} 3_{2} \rightarrow 2_{1}$ & $\mathrm{SO}_{6} \rightarrow 5_{4}$ & ${ }^{34} \mathrm{SO} 3_{2} \rightarrow 2_{1}$ & \multicolumn{2}{|c|}{$\mathrm{SO}_{2} 5_{1,5} \rightarrow 4_{0,4}$} & \multicolumn{2}{|c|}{$\mathrm{H}_{2} \mathrm{~S} 2_{2,0} \rightarrow 2_{1,1}$} & $\mathrm{H}_{2} \mathrm{~S}_{1,0} \rightarrow 1_{0,1}$ \\
\hline$v(\mathrm{GHz})$ & 99.299 & 219.949 & 97.715 & 135.696 & & 216.710 & & 168.762 \\
\hline$E_{\text {up }} / \mathrm{k}(\mathrm{K})$ & 9 & 35 & 9 & 15.6 & & 84 & & 24 \\
\hline$n_{\mathrm{cr}}\left(\mathrm{cm}^{-3}\right)$ & $10^{5}$ & $10^{6}$ & $10^{5}$ & $2 \times 10^{6}$ & & $10^{6}$ & & $2 \times 10^{6}$ \\
\hline Telescope & SEST & SEST & IRAM & IRAM & IRAM & SEST & IRAM & IRAM \\
\hline$T_{\text {sys }}(\mathrm{K})$ & 160 & 250 & 140 & 400 & 270 & 220 & 600 & 700 \\
\hline Beam (") & 51 & 24 & 26 & 18 & 18 & 25 & 11 & 14.5 \\
\hline$B_{\text {eff }}$ & 0.75 & 0.5 & 0.75 & 0.59 & 0.71 & 0.5 & 0.57 & 0.65 \\
\hline$\Delta v(\mathrm{kHz})$ & 86 & 86 & 40 & 39 & 39 & 86 & 42 & 42 \\
\hline$\Delta \alpha \times \Delta \delta\left("{ }^{\prime \prime} \times{ }^{\prime \prime}\right)$ & $280 \times 190$ & $288 \times 216$ & - & $120 \times 100$ & - & $120 \times 100$ & - & - \\
\hline sampling $\left({ }^{\prime \prime}\right)$ & 24 & 24 & - & 12 & - & 24 & - & - \\
\hline
\end{tabular}

source morphologies. Both with the IRAM and SEST telescopes, pointing and focus was checked every $2 \mathrm{~h}$, using planets, maser sources and strong extragalactic continuum sources. The pointing corrections were found to be always smaller than $3^{\prime \prime}$ and 5" with the IRAM and SEST telescopes respectively. Polynomial baselines of order 3 or less have been subtracted from the spectra. The observing parameters for both telescopes are listed in Table 1. In the following, all intensities will be given in main-beam brightness temperatures. Below we give some information which are specific to the IRAM-30m and SEST observations.

IRAM observations: the IRAM $30-\mathrm{m}$ telescope is located at an altitude of $2920 \mathrm{~m}$ near the summit of Pico Veleta in Southern Spain. The ${ }^{34} \mathrm{SO} 3_{2} \rightarrow 2_{1}, \mathrm{SO}_{2}$ and $\mathrm{H}_{2} \mathrm{~S}$ molecular emissions were observed simultaneously with the 3 , 2 and $1 \mathrm{~mm}$ SIS receivers respectively, available at the IRAM-30m. The $\mathrm{SO}_{2}$ emission observed in June 1997 was observed simultaneously with other molecular transitions not presented here. The image sideband rejections of all receivers were always higher than $10 \mathrm{~dB}$. Typical system temperatures were in the range $T_{\text {sys }} \sim 300-600 \mathrm{~K}$. The three receivers were connected to units of the autocorrelator, set to provide spectral resolutions of 40,40 , and $80 \mathrm{kHz}$ at 98,136 , and $217 \mathrm{GHz}$ respectively. At those frequencies, the velocity resolutions are of the order of $0.1-0.2 \mathrm{~km} \mathrm{~s}^{-1}$.

SEST observations: the SEST telescope is a $15-\mathrm{m}$ single dish millimeter telescope operated jointly by ESO and a consortium of Swedish institutions. It is located at an altitude of about $2450 \mathrm{~m}$ in La Silla (Chile). To obtain the SO and $\mathrm{H}_{2} \mathrm{~S}$ maps shown here, we used the dual $3 / 1.3 \mathrm{~mm}$ receiver. System temperatures were in the range $T_{\text {sys }} \sim 300-500 \mathrm{~K}$. This receiver was always connected to the high resolution acousto-optical spectrometer available at SEST, which provides 2048 channel of $43 \mathrm{kHz}$ width giving a $86 \mathrm{kHz}$ spectral resolution. At the frequencies considered here, that corresponds to a velocity resolution of $0.1-0.2 \mathrm{~km} \mathrm{~s}^{-1}$, comparable to that of the IRAM data.

\section{Results}

Figure 2 shows the velocity-integrated intensity maps of the SO $3_{2} \rightarrow 2_{1}$, SO $6_{5} \rightarrow 5_{4}$ and $\mathrm{SO}_{2} 5_{1,5} \rightarrow 4_{0,4}$ lines. The spectra (not smoothed) of the six transitions of Table 1 observed towards the "key" positions are displayed in Fig. 3, and the relevant line parameters are reported in Table 2 . The $\mathrm{SiO} 2 \rightarrow 1 \mathrm{ob}-$ servations, published by CCLCL01, have been added in Fig. 3 and in Table 2 for comparisons.

The main-beam brightness peak temperature $T_{\mathrm{MB}}$, and the linewidth $\Delta v(F W H M)$ of Table 2 were estimated from a Gaussian fit to the whole line profile, even in the case where profiles are double-peaked. We also estimated the width of the equivalent Gaussian with the same integrated and peak intensity as the observed line and found that the two methods differ by less than $30 \%$ with the exception of the SO $6_{5} \rightarrow 5_{4}$ line in $\mathrm{W} 1$ which has an equivalent width of $2.5 \mathrm{~km} \mathrm{~s}^{-1}$ (versus $1.3 \mathrm{~km} \mathrm{~s}^{-1}$ in Table 2). On the contrary, the velocityintegrated intensity is the area integrated in the interval of velocities at the "zero-intensity" level. The velocity $v_{\text {LSR }}$ is the velocity at the peak or at the absorption dip for double-peaked profiles. They are roughly the same for each observed transition and position, with the exception of E2(HVC), whose emission is red-shifted by $\sim 6 \mathrm{~km} \mathrm{~s}^{-1}$.

The first remarkable result is that, against probably naive expectations, the shocked regions are not necessarily associated with evident enhancements of the emission of the observed SO, $\mathrm{SO}_{2}$ and $\mathrm{H}_{2} \mathrm{~S}$ lines. For example, the E2 region, the brightest $\mathrm{SiO} 2-1$ emission peak (see CCLCL01) is undetected in $\mathrm{SO}_{2}$, and only marginally detected in the lowest SO transition.

The second result is that IRAS 16293 is the only site where all the molecular lines have been detected, including the highenergy transition $\mathrm{H}_{2} \mathrm{~S} 2_{2,0} \rightarrow 2_{1,1}$. Quite interestingly, we find that not only the line profiles are broader than in the cloud but their widths tend to increase with the upper energy level of the transition. We will discuss in the Sect. 5 the implication of this behavior. 

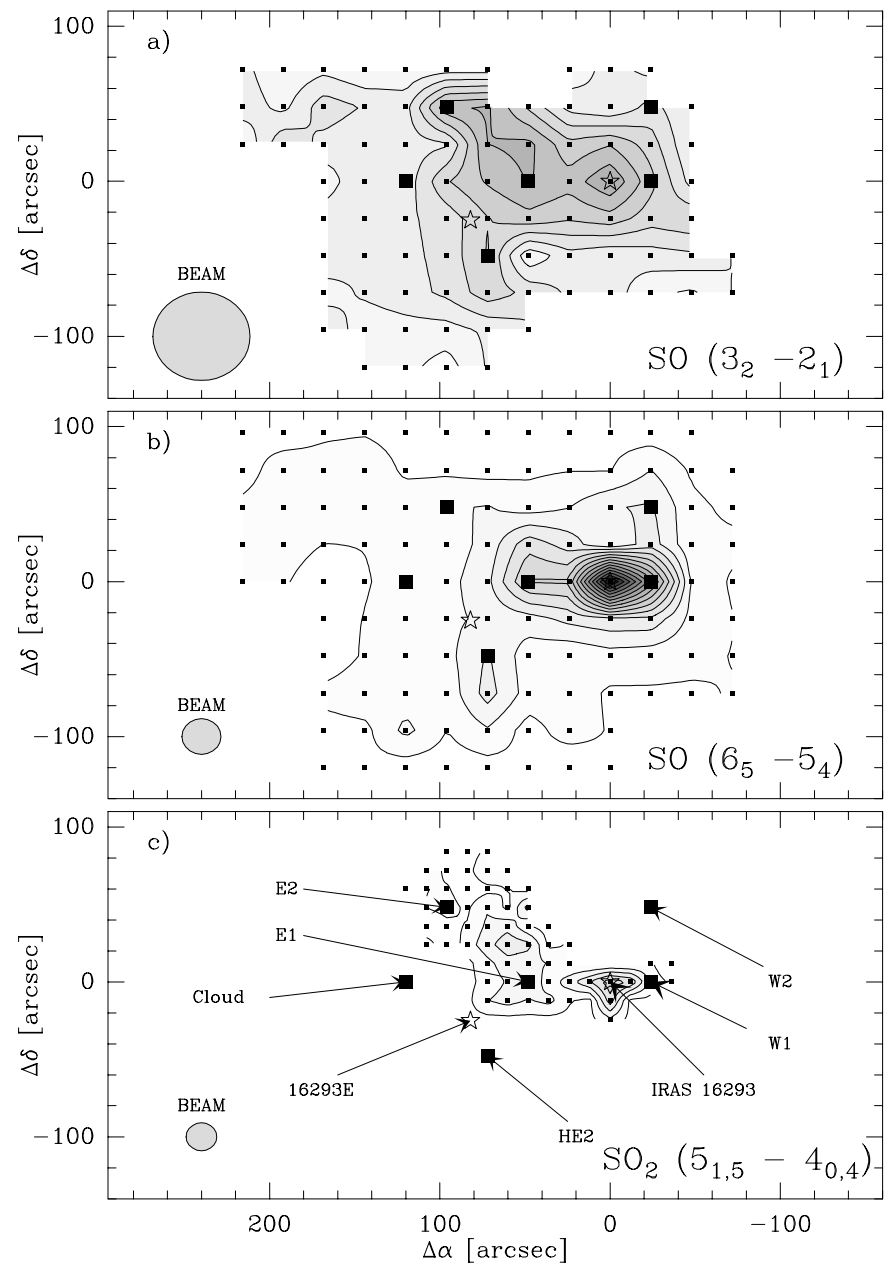

Fig. 2. Integrated intensity maps of SO $3_{2} \rightarrow 2_{1}$ a), SO $6_{5} \rightarrow 5_{4}$ b) and $\mathrm{SO}_{2} 5_{1,5} \rightarrow 4_{0,4}$ c). a) and b): first level is $0.5 \mathrm{~K} \mathrm{~km} \mathrm{~s}^{-1}$ with level step of $1.0 \mathrm{~K} \mathrm{~km} \mathrm{~s}^{-1}$. c): first level is $0.3 \mathrm{~K} \mathrm{~km} \mathrm{~s}^{-1}$ with level step of $1.0 \mathrm{~K} \mathrm{~km} \mathrm{~s}^{-1}$. In each map, the black points represent the observed positions. The two stars symbols show the position of the two protostellar sources. The arrows point to the eight "key" positions, whose spectra are displayed in Fig. 3.

We now discuss in more detail the results toward the eight "key" positions.

\subsection{SO emission}

The SO $3_{2} \rightarrow 2_{1}$ emission is relatively bright $(\sim 3 \mathrm{~K})$ all over the cloud (Figs. 2 and 3 ). All the line profiles are relatively narrow (with linewidths ranging between 1 and $2 \mathrm{~km} \mathrm{~s}^{-1}$ ) and peak around the systemic cloud velocity at $v_{\mathrm{LSR}}=3.9 \mathrm{~km} \mathrm{~s}^{-1}$. The absorption dip around the systemic velocity seen towards IRAS 16293 is probably due to the absorption by foreground material, as also seen in the $\mathrm{H}_{2} \mathrm{CO}$ transitions (Loinard et al. 2001). On the other hand, the absorption dip seen in the ${ }^{34} \mathrm{SO}$ towards W1 may point to a very large SO column density in that direction or two different kinematic components. The contrast of the velocity-integrated SO molecular emission at the various positions in the cloud is relatively low: the emission peaks towards IRAS 16293 but otherwise it is widespread in the region between W1 and E2 (Fig. 2).
Remarkably, there is not a much brighter SO $3_{2} \rightarrow 2_{1}$ emission towards the shocked regions with respect to the molecular cloud. The only two clear exceptions are represented by E1 and E2, where a high velocity component appears in the low energy SO transition (Fig. 3). In the following we will analyze separately the E2 low velocity component centered at $3.9 \mathrm{~km} \mathrm{~s}^{-1}$ (hereinafter E2(LVC)), and the E2 high velocity component at $10 \mathrm{~km} \mathrm{~s}^{-1}$ (hereinafter E2(HVC)), because the two components can be clearly disentangled. On the contrary, in E1 the situation is less clear and hence we didn't pursue a separated analysis of the two components. We emphasize that the E2(HVC) component is detected at more than 3 RMS all along the red lobe of the outflow and it is also observed in the $\mathrm{SiO}$ transitions (CCLCL01). This high velocity component is not seen in $\mathrm{SO}_{6} \rightarrow 5_{4}$ probably because of unfavorable excitation conditions. Indeed, we will show in the next section that the gas density in E2(HVC) is lower than in the ambient gas (see Table 3). This fact, already recognized by CCLCL01, leads to the supposition that the E2(HVC) may represent the wind shock, whereas the lower velocity component, E2(LVC), may trace the so called cloud shock (Hollenbach 1998). Finally, we also see hints of red and blue wings originating from the molecular outflows driven either by IRAS 16293 or 16293E in the direction of W1, E1 and HE2 whereas no wing is seen in W2.

In the higher energy line SO $6_{5} \rightarrow 5_{4}$ the emission is peaked towards IRAS 16293 and W1 (cf. Fig. 2) while the emission from the cloud is, as expected, weak. Relatively strong emission is also detected around E1. Moreover, the SO $65 \rightarrow 5_{4}$ spectra in those positions have pronounced red and blue wings. The same happens in HE2 where the SO $6_{5} \rightarrow 5_{4}$ spectrum shows clearly the presence of a blue wing. The emitting region around IRAS 16293 has a characteristic size of $\alpha \times \delta=39^{\prime \prime} \times 16^{\prime \prime}$ namely it is not resolved in $\delta$. Finally, the observed SO spectra towards 16293E are similar to those in the cloud: narrow, intense in the $\mathrm{SO} 3_{2} \rightarrow 2_{1}$ transition and weak in the SO $6_{5} \rightarrow 5_{4}$ transition, and no signs of wings are evident. Overall, 16293E does not seem to have any enhanced SO emission compared with the cloud.

\section{2. $\mathrm{SO}_{2}$ and $\mathrm{H}_{2} \mathrm{~S}$ emission}

Unlike the SO $3_{2} \rightarrow 2_{1}$, the $\mathrm{SO}_{2} 5_{1,5} \rightarrow 4_{0,4}$ emission is limited to a few spots (see Figs. 2 and 3), specifically toward IRAS 16293, E1 and W2, probably because of the excitation conditions (the critical density of the $\mathrm{SO}_{2} 5_{1,5} \rightarrow 4_{0,4}$ transition is about 20 times the $\mathrm{SO} 3_{2} \rightarrow 2_{1}$ critical density). Unfortunately, because of the lack of other observations around W2 we have no idea of its extent. Remarkably, no significant $\mathrm{SO}_{2}$ emission is detected towards $\mathrm{E} 2 . \mathrm{SO}_{2}$ has also been detected at the cloud position. The linewidth $\left(1.4 \mathrm{~km} \mathrm{~s}^{-1}\right)$ is equivalent to that of $\mathrm{N}_{2} \mathrm{H}^{+} 1 \rightarrow 0-$ a good tracer of extended, cold and quiescent gas - observed at the same position (CCLCL01).

Probably because of similar critical densities, the $\mathrm{SO}_{2} 5_{1,5} \rightarrow 4_{0,4}$ and $\mathrm{SO} 6_{5} \rightarrow 5_{4}$ emission shows up in the same regions. In addition, with the exception of E1, the $\mathrm{SO}_{2} 5_{1,5} \rightarrow 4_{0,4}$ spectra show characteristics similar to the 

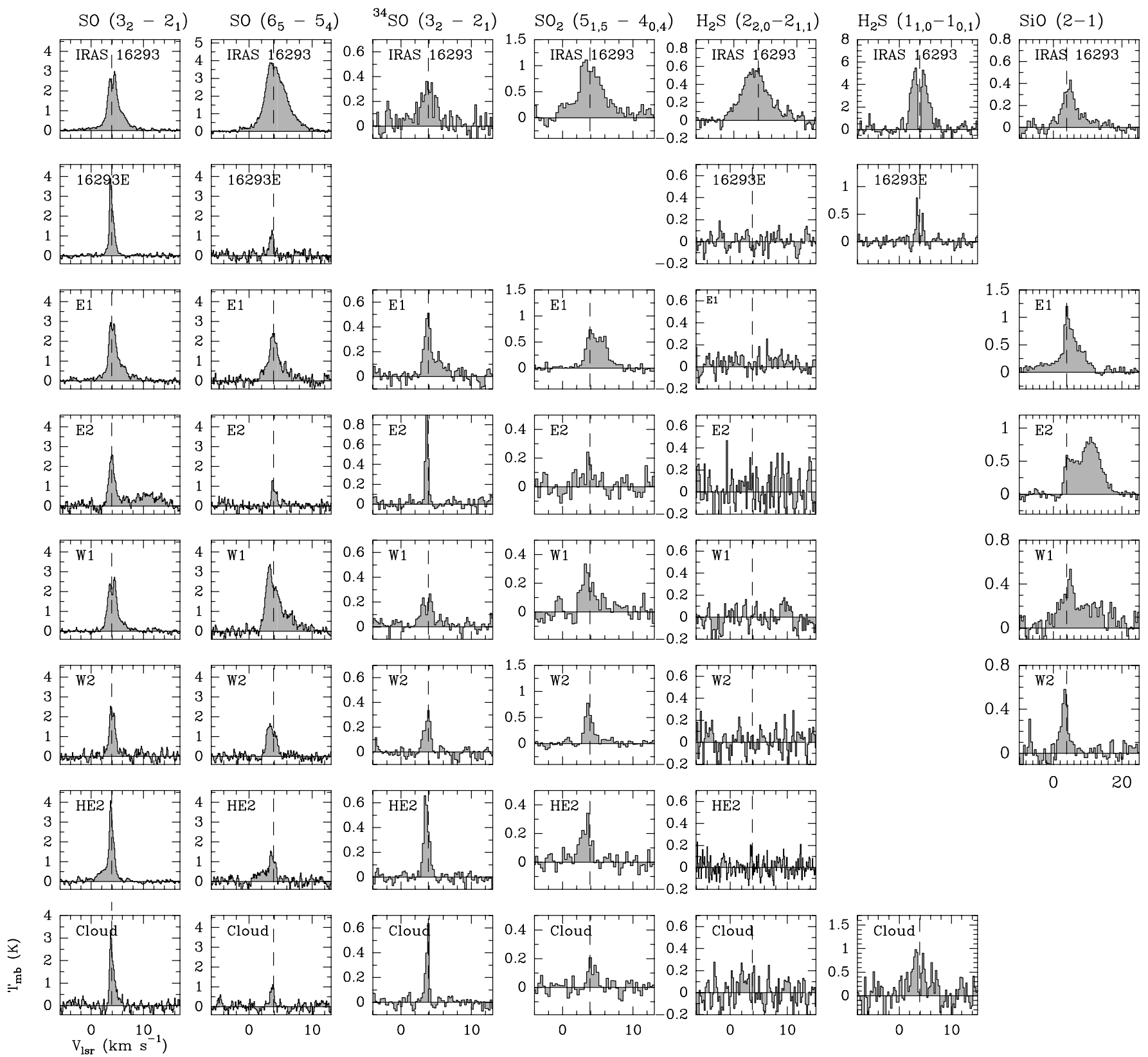

Fig. 3. Spectra (not smoothed) of SO $3_{2} \rightarrow 2_{1}$, SO $6_{5} \rightarrow 5_{4},{ }^{34} \mathrm{SO}_{2} \rightarrow 2_{1}, \mathrm{SO}_{2} 5_{1,5} \rightarrow 4_{0,4}, \mathrm{H}_{2} \mathrm{~S} 2_{2,0} \rightarrow 2_{1,1}, \mathrm{H}_{2} \mathrm{~S} 1_{1,0} \rightarrow 1_{0,1}$ and $\mathrm{SiO} 2 \rightarrow 1$ at "key" points (see text) in the L1689N molecular cloud. The vertical dashed line on each spectrum shows the position of the cloud systemic velocity $\left(v_{\mathrm{LSR}}=3.9 \mathrm{~km} \mathrm{~s}^{-1}\right)$.

SO $6_{5} \rightarrow 5_{4}$ spectra, like for example the linewidth, in all "key" positions. On the contrary, in E1 the SO line profiles are characterized by a strong ambient component with blue- and redshifted wings of much lower brightness, whereas the highvelocity component appears as bright as the ambient component in the $\mathrm{SO}_{2}$ line, so that the whole $\mathrm{SO}_{2} 5_{1,5} \rightarrow 4_{0,4}$ linewidth is approximately twice that of the SO $6_{5} \rightarrow 5_{4}$.

IRAS 16293 is the only position where $\mathrm{H}_{2} \mathrm{~S} 2_{2,0} \rightarrow 2_{1,1}$ has been detected. The line is relatively bright and rather broad $\left(\Delta v=5.0 \mathrm{~km} \mathrm{~s}^{-1}\right)$. There is no emission detected in shocks nor in the ambient cloud. Conversely, the lower $\mathrm{H}_{2} \mathrm{~S}$ transition, observed only toward IRAS 16293, 16293E and the cloud, has been detected in all these positions with a double-peaked profile towards the protostars and possibly the cloud position. The absorption dip is likely due to the optical depth of the line. In fact, Minh et al. (1991) have observed the same $\mathrm{H}_{2} \mathrm{~S}$ transition towards several star forming regions and found an opacity of $\sim 10$ for similar column densities.

\subsection{Comparison with $\mathrm{SiO}$}

Contrary to the sulphur bearing species, the emission of SiO $2 \rightarrow 1$ is stronger in the shocked regions than towards IRAS 16293. The spectra in E1 and W1 show high velocity 
Table 2. Line parameters $\left(T_{\mathrm{MB}}(\mathrm{K}), \Delta v\left(\mathrm{~km} \mathrm{~s}^{-1}\right), v_{\mathrm{LSR}}\left(\mathrm{km} \mathrm{s}^{-1}\right), \int T_{\mathrm{MB}} \delta v\left(\mathrm{~K} \mathrm{~km} \mathrm{~s}^{-1}\right)\right)$ for the various transitions observed at the "key" positions: the two protostars IRAS 16293 and 16293E, the shocked regions E1, E2, W1, W2 and HE2, and the reference position in the cloud. The $\mathrm{SiO}$ observations were previously published in CCLCL01. When no signal is detected, we give an upper limit for the intensity equal to $3 \mathrm{RMS}$ of the relevant spectrum. The parameters are taken from the spectra not smoothed. The symbol "-" implies that the relevant transition has not been observed or detected.

\begin{tabular}{|c|c|c|c|c|c|c|c|c|}
\hline & & $\begin{array}{c}\text { SO } \\
3_{2} \rightarrow 2_{1} \\
\end{array}$ & $\begin{array}{c}\text { SO } \\
6_{5} \rightarrow 5_{4} \\
\end{array}$ & $\begin{array}{c}{ }^{34} \mathrm{SO} \\
3_{2} \rightarrow 2_{1} \\
\end{array}$ & $\begin{array}{c}\mathrm{SO}_{2} \\
5_{1,5} \rightarrow 4_{0,4}\end{array}$ & $\begin{array}{c}\mathrm{H}_{2} \mathrm{~S} \\
2_{2,0} \rightarrow 2_{1,1}\end{array}$ & $\begin{array}{c}\mathrm{H}_{2} \mathrm{~S} \\
1_{1,0} \rightarrow 1_{0,1}\end{array}$ & $\begin{array}{c}\mathrm{SiO} \\
2 \rightarrow 1 \\
\end{array}$ \\
\hline IRAS & $T_{\mathrm{MB}}$ & $2.9 \pm 0.1$ & $3.7 \pm 0.1$ & $0.3 \pm 0.1$ & $1.1 \pm 0.1$ & $0.5 \pm 0.04$ & $6.2 \pm 0.6$ & $0.35 \pm 0.05$ \\
\hline \multirow[t]{4}{*}{$16293-2422$} & $\Delta v$ & $2.0 \pm 0.1$ & $3.9 \pm 0.0$ & $2.8 \pm 0.5$ & $4.2 \pm 0.2$ & $5.0 \pm 0.2$ & $3.0 \pm 0.0$ & $5.0 \pm 0.4$ \\
\hline & $v_{\mathrm{LSR}}$ & 4.0 & 3.9 & 3.9 & 3.9 & 3.3 & 3.9 & 4.2 \\
\hline & $\int T_{\mathrm{MB}} \delta v$ & $8.0 \pm 1.2$ & $16.0 \pm 2.5$ & $1.0 \pm 0.3$ & $4.6 \pm 0.9$ & $3.0 \pm 0.5$ & $17.5 \pm 3.2$ & $1.9 \pm 0.1$ \\
\hline & $T_{\mathrm{MB}}$ & $3.4 \pm 0.1$ & $1.2 \pm 0.2$ & - & - & $\leq 0.2$ & $1.1 \pm 0.1$ & - \\
\hline \multirow[t]{4}{*}{$16293 \mathrm{E}$} & $\Delta v$ & $1.0 \pm 0.0$ & $0.8 \pm 0.1$ & - & - & - & $1.2 \pm 0.1$ & - \\
\hline & $v_{\mathrm{LSR}}$ & 3.7 & 3.7 & - & - & - & 3.9 & - \\
\hline & $\int T_{\mathrm{MB}} \delta v$ & $3.8 \pm 0.6$ & $1.0 \pm 0.2$ & - & - & $\leq 0.1$ & $0.6 \pm 0.1$ & - \\
\hline & $T_{\mathrm{MB}}$ & $2.9 \pm 0.1$ & $2.4 \pm 0.2$ & $0.5 \pm 0.1$ & $0.7 \pm 0.1$ & $\leq 0.2$ & - & $1.1 \pm 0.1$ \\
\hline \multirow[t]{3}{*}{ E1 } & $\Delta v$ & $1.8 \pm 0.2$ & $1.6 \pm 0.1$ & $1.2 \pm 0.1$ & $3.1 \pm 0.1$ & - & - & $2.8 \pm 0.1$ \\
\hline & $v_{\mathrm{LSR}}$ & 4.0 & 3.8 & 3.9 & 4.0 & - & - & 3.6 \\
\hline & $\int T_{\mathrm{MB}} \delta v$ & $7.3 \pm 1.2$ & $5.6 \pm 1.0$ & $0.8 \pm 0.2$ & $2.2 \pm 0.4$ & $\leq 0.3$ & - & $3.3 \pm 0.3$ \\
\hline $\mathrm{E} 2$ & $T_{\mathrm{MB}}$ & $2.4 \pm 0.2$ & $1.1 \pm 0.2$ & $0.9 \pm 0.04$ & $\leq 0.2$ & $\leq 0.4$ & - & $0.53 \pm 0.06$ \\
\hline \multirow[t]{3}{*}{ LVC } & $\Delta v$ & $1.3 \pm 0.1$ & $0.9 \pm 0.1$ & $0.5 \pm 0.02$ & - & - & - & $3.2 \pm 0.1$ \\
\hline & $v_{\mathrm{LSR}}$ & 3.9 & 3.8 & 3.6 & - & - & - & 4.0 \\
\hline & $\int T_{\mathrm{MB}} \delta v$ & $3.7 \pm 0.7$ & $1.0 \pm 0.2$ & $0.5 \pm 0.1$ & $\leq 0.1$ & $\leq 0.4$ & - & $1.8 \pm 0.1$ \\
\hline E2 & $T_{\mathrm{MB}}$ & $0.5 \pm 0.15$ & $\leq 0.6$ & $\leq 0.1$ & $\leq 0.2$ & $\leq 0.4$ & - & $0.83 \pm 0.06$ \\
\hline \multirow[t]{4}{*}{ HVC } & $\Delta v$ & $6.6 \pm 0.6$ & - & - & - & - & - & $6.3 \pm 0.2$ \\
\hline & $v_{\mathrm{LSR}}$ & 10.4 & - & - & - & - & - & 11 \\
\hline & $\int T_{\mathrm{MB}} \delta v$ & $3.6 \pm 0.7$ & $\leq 0.5$ & $\leq 0.2$ & $\leq 0.3$ & $\leq 0.8$ & - & $5.6 \pm 0.2$ \\
\hline & $T_{\mathrm{MB}}$ & $2.6 \pm 0.1$ & $3.3 \pm 0.2$ & $0.2 \pm 0.04$ & $0.3 \pm 0.1$ & $\leq 0.2$ & - & $0.3 \pm 0.1$ \\
\hline \multirow[t]{4}{*}{ W1 } & $\Delta v$ & $1.8 \pm 0.2$ & $1.3 \pm 0.03$ & $2.2 \pm 0.2$ & $1.9 \pm 0.3$ & - & - & $3.3 \pm 0.8$ \\
\hline & $v_{\mathrm{LSR}}$ & 4.0 & 3.3 & 3.7 & 3.3 & - & - & 4.6 \\
\hline & $\int T_{\mathrm{MB}} \delta v$ & $5.8 \pm 1.0$ & $9.0 \pm 1.5$ & $0.5 \pm 0.1$ & $0.7 \pm 0.2$ & $\leq 0.4$ & - & $1.1 \pm 0.2$ \\
\hline & $T_{\mathrm{MB}}$ & $2.4 \pm 0.2$ & $1.6 \pm 0.2$ & $0.30 \pm 0.04$ & $0.7 \pm 0.1$ & $\leq 0.2$ & - & $0.5 \pm 0.1$ \\
\hline \multirow[t]{4}{*}{ W2 } & $\Delta v$ & $1.4 \pm 0.1$ & $1.6 \pm 0.1$ & $1.1 \pm 0.1$ & $1.2 \pm 0.1$ & - & - & $2.1 \pm 0.3$ \\
\hline & $v_{\mathrm{LSR}}$ & 3.8 & 3.3 & 3.9 & 3.7 & - & - & 3.5 \\
\hline & $\int T_{\mathrm{MB}} \delta v$ & $3.8 \pm 0.7$ & $2.7 \pm 0.5$ & $0.3 \pm 0.1$ & $0.9 \pm 0.2$ & $\leq 0.3$ & - & $1.2 \pm 0.1$ \\
\hline & $T_{\mathrm{MB}}$ & $3.7 \pm 0.1$ & $1.4 \pm 0.2$ & $0.6 \pm 0.04$ & $0.3 \pm 0.04$ & $\leq 0.2$ & - & - \\
\hline \multirow[t]{4}{*}{ HE2 } & $\Delta v$ & $0.9 \pm 0.1$ & $0.9 \pm 0.1$ & $0.9 \pm 0.1$ & $1.8 \pm 0.02$ & - & - & - \\
\hline & $v_{\mathrm{LSR}}$ & 3.7 & 3.5 & 3.5 & 3.6 & - & - & - \\
\hline & $\int T_{\mathrm{MB}} \delta v$ & $5.3 \pm 0.8$ & $2.7 \pm 0.5$ & $0.6 \pm 0.1$ & $0.6 \pm 0.1$ & $\leq 0.3$ & - & - \\
\hline & $T_{\mathrm{MB}}$ & $3.1 \pm 0.2$ & $1.1 \pm 0.2$ & $0.6 \pm 0.04$ & $0.2 \pm 0.04$ & $\leq 0.3$ & $0.8 \pm 0.2$ & - \\
\hline \multirow[t]{3}{*}{ Cloud } & $\Delta v$ & $1.0 \pm 0.04$ & $0.7 \pm 0.1$ & $0.5 \pm 0.1$ & $1.4 \pm 0.2$ & - & $1.1 \pm 0.4$ & - \\
\hline & $v_{\mathrm{LSR}}$ & 3.8 & 3.8 & 3.8 & 3.9 & - & 3.4 & - \\
\hline & $\int T_{\mathrm{MB}} \delta v$ & $3.2 \pm 0.6$ & $0.8 \pm 0.2$ & $0.3 \pm 0.1$ & $0.2 \pm 0.1$ & $\leq 0.3$ & $1.0 \pm 0.2$ & - \\
\hline
\end{tabular}

wings much more marked than for the $\mathrm{SO}$ and $\mathrm{SO}_{2}$ lines. The high velocity component of E2 seen in SO $3_{2} \rightarrow 2_{1}$ is very strong in $\mathrm{SiO} 2 \rightarrow 1$ and have similar $\Delta v\left(\sim 6.5 \mathrm{~km} \mathrm{~s}^{-1}\right)$ and $v_{\mathrm{LSR}}\left(\sim 10 \mathrm{~km} \mathrm{~s}^{-1}\right)$ for these two transitions.

\section{Column densities}

In this section we estimate the column densities of the observed species, namely $\mathrm{SO}, \mathrm{SO}_{2}$ and $\mathrm{H}_{2} \mathrm{~S}$, as well of the $\mathrm{SiO}$ and $\mathrm{H}_{2} \mathrm{CO}$ previously observed by CCLCLO1, in the "key" 
positions. In order to do that, we first estimate the density and temperature of the gas in each position. To derive the gas temperature and density, as well as the column densities of the different species, we used the theoretical predictions from an LVG (Large Velocity Gradient) model described in detail in the next paragraph. The next three paragraphs describe the derivation of the gas temperature and densities as well as the column density of the observed species in the cloud, in the shocked regions, and in IRAS 16293 respectively. Note that we did not carry out the analysis on 16293E, because the observed emission in that position seems to be dominated by the cloud emission.

\subsection{LVG model description}

To derive the gas temperature and density, as well as the column densities, we compared the observed line intensities with the theoretical predictions from a LVG model, which selfconsistently accounts for the excitation conditions (density and temperature) as well as the line opacities. For the escape probability $\beta$ we used the following function of the line optical depth: $\beta=\frac{1-\exp [-3 \tau]}{3 \tau}$, valid in the case of a homogeneous and isothermal semi-infinite slab. More details can be found in Ceccarelli et al. (2002). Note that the dust emission is neglected in the present computations.

The spectroscopic data of $\mathrm{SO}, \quad \mathrm{SO}_{2}, \mathrm{H}_{2} \mathrm{~S}, \quad \mathrm{SiO}$ and $\mathrm{H}_{2} \mathrm{CO}$ are all taken from the JPL catalogue (http://spec.jpl.nasa.gov/ftp/pub/catalog/

catform.html; Pickett et al. 1998). The collisional coefficients are from Green (1994) for SO, Palma (1987) for $\mathrm{SO}_{2}$, Turner et al. (1992) for $\mathrm{SiO}$, and Green (1991) for $\mathrm{H}_{2} \mathrm{CO}$ respectively. The collisional coefficients of $\mathrm{H}_{2} \mathrm{~S}$ are not available in literature, and we estimated them from the collisional coefficients of $\mathrm{H}_{2} \mathrm{O}$, multiplied by a factor 5 (following the discussion in Turner 1996). This is a very rough approximation, giving rise to unfortunately very rough estimates of the $\mathrm{H}_{2} \mathrm{~S}$ column density. Furthermore, the collisional coefficients used for the other molecules are not available in the full range of temperatures probed by our observations. In particular the low temperature regime is often missing. In this case we have extrapolated the coefficients at the lowest available temperature with a $\sqrt{T_{\text {kin }}}$ law. Finally, our code considers the first 50 rotational levels of each molecule, unless the relevant collisional coefficients are available for a lower number of levels.

\subsection{Cloud}

To estimate the gas density and temperature, and the SO column density in the cloud reference position we simultaneously best-fitted the ${ }^{34} \mathrm{SO}$ and ${ }^{32} \mathrm{SO} 3_{2} \rightarrow 2_{1}$ observations (that give a line opacity for ${ }^{32} \mathrm{SO} \tau \sim 1.5$ ), and the ${ }^{32} \mathrm{SO} 3_{2} \rightarrow 2_{1}$ and $6_{5} \rightarrow$ $5_{4}$ observations (whose ratio, together with the ${ }^{32} \mathrm{SO} 3_{2} \rightarrow$ $2{ }_{1}$ intensity, gives pairs of gas temperature and density values). Note that we used the elemental ratio ${ }^{34} \mathrm{~S} /{ }^{32} \mathrm{~S}=22.5$ (Wilson \& Rood 1994; Chin et al. 1996; Lucas \& Liszt 1998), and we assumed that the emission fills up the beam, when comparing observations obtained with different telescopes. The result of
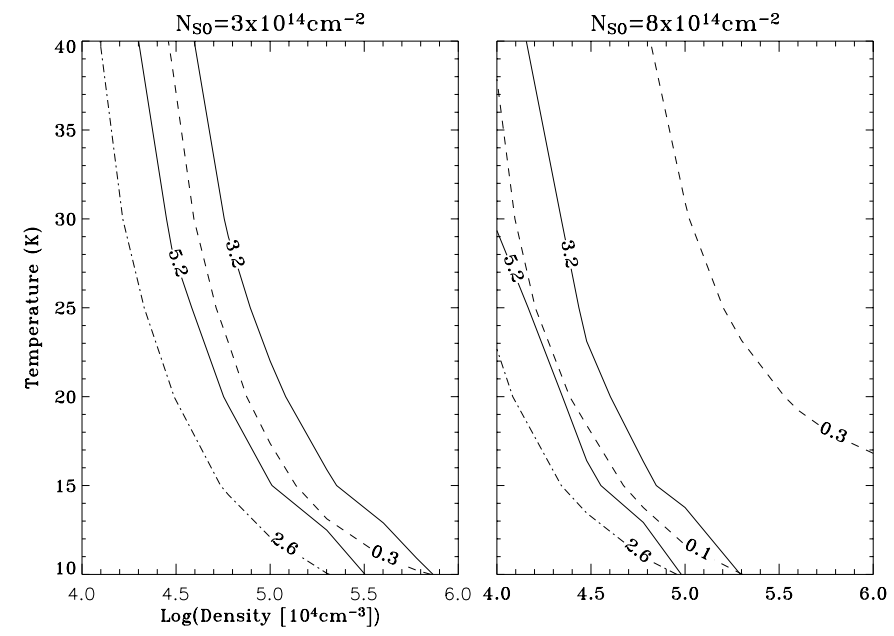

Fig. 4. Cloud reference position modeling. Theoretical predictions as function of the gas temperature and density for two different SO column densities. The curves refer the values of three observed quantities taking into account the uncertainties. Solid lines: observed integrated intensity ratio SO $3_{2} \rightarrow 2_{1}$ over SO $6_{5} \rightarrow 5_{4}$, between 3.2 and 5.2. Dashed lines: escape probability, derived from the ${ }^{34} \mathrm{SO}$ and ${ }^{32} \mathrm{SO} 3_{2} \rightarrow 2_{1}$ line ratio, between 0.1 and 0.3 . Dot-dashed line: the minimum integrated intensity of the $\mathrm{SO} 3_{2} \rightarrow 2_{1}$ transition, $2.6 \mathrm{~K} \mathrm{~km} \mathrm{~s}^{-1}$. Note that the intensity increases with increasing temperature, i.e. towards the upper part of the panel. Analogously, the lower limit of the escape probability $(0.1)$ in the left panel is not shown because it lies outside the plot.

the modeling is shown in Fig. 4. We found that the ensemble of our observations constrains the SO column density in the cloud to be in the range 3 to $8 \times 10^{14} \mathrm{~cm}^{-2}$. Adopting the gas temperature of $26 \mathrm{~K}$, as found by Caux et al. (1999), we derive a density of $3 \times 10^{4} \mathrm{~cm}^{-3}$.

The column densities of the other species, namely $\mathrm{SO}_{2}$ and $\mathrm{H}_{2} \mathrm{~S}$, have been derived assuming the same density and temperature. The $\mathrm{SO}_{2}$ abundance was found to be $4 \times 10^{-8}$, a value equivalent to the ones obtained in other clouds (see Table 4). In the case of $\mathrm{H}_{2} \mathrm{~S}$ we cross-checked the column density derivation with LTE computations, because of the rough approximation of the collisional coefficients. For this we assumed an excitation temperature of $5 \mathrm{~K}$, as found by Swade (1989) and Hirahara et al. (1995) in similar molecular clouds (L134N and TMC-1) for several molecules whose fundamental transitions have $A$-coefficients similar to that for the $\mathrm{H}_{2} \mathrm{~S} 1_{1,0} \rightarrow 1_{0,1}$ transition. In fact Minh et al. (1989) used an excitation temperature of $5 \mathrm{~K}$ for the $\mathrm{H}_{2} \mathrm{~S} 1_{1,0} \rightarrow 1_{0,1}$ transition to compute the column density of $\mathrm{H}_{2} \mathrm{~S}$ in the molecular clouds L134N and TMC-1, which have temperatures and densities similar to L1689N. Note that increasing the excitation temperature by a factor two, would decrease the $\mathrm{H}_{2} \mathrm{~S}$ column density by the same amount. We assumed a $\mathrm{H}_{2} \mathrm{~S}$ ortho-to-para ratio equal to 3 . Finally the $\mathrm{SiO}$ and $\mathrm{H}_{2} \mathrm{CO}$ column densities are taken from the modeling by Ceccarelli et al. $(2000 \mathrm{~b}, 2001)$ 
Table 3. Gas temperature and density, and column densities of $\mathrm{SO}, \mathrm{SO}_{2}, \mathrm{H}_{2} \mathrm{~S}, \mathrm{SiO}$ and $\mathrm{H}_{2} \mathrm{CO}$ in six "key" positions. The column densities are beam-averaged in the shocked regions (on 27" for $\mathrm{SiO}, 26^{\prime \prime}$ for $\mathrm{H}_{2} \mathrm{CO}, 24^{\prime \prime}$ for $\mathrm{SO}, 18^{\prime \prime}$ for $\mathrm{SO}_{2}$ and $25^{\prime \prime}$ for $\mathrm{H}_{2} \mathrm{~S}$ ), whereas they are corrected from the beam dilution in IRAS 16293. Errors on the estimates are around 15\% if considering only the statistical uncertainties. However, a factor two of uncertainty has to be considered, when the uncertainty on the density and temperature of the emitting gas is considered.

\begin{tabular}{c|cc|ccccc}
\hline \hline & $\begin{array}{c}\text { Density } \\
\left(10^{5} \mathrm{~cm}^{-3}\right)\end{array}$ & $\begin{array}{c}T \\
(\mathrm{~K})\end{array}$ & $\begin{array}{c}N_{\mathrm{SO}} \\
\left(10^{13} \mathrm{~cm}^{-2}\right)\end{array}$ & $\begin{array}{c}N_{\mathrm{SO}_{2}} \\
\left(10^{13} \mathrm{~cm}^{-2}\right)\end{array}$ & $\begin{array}{c}N_{\mathrm{H}_{2} \mathrm{~S}} \\
\left(10^{13} \mathrm{~cm}^{-2}\right)\end{array}$ & $\begin{array}{c}N_{\mathrm{SiO}_{2}} \\
\left(10^{13} \mathrm{~cm}^{-2}\right)\end{array}$ & $\begin{array}{c}N_{\mathrm{H}_{2} \mathrm{CO}} \\
\left(10^{13} \mathrm{~cm}^{-2}\right)\end{array}$ \\
\hline IRAS 16293 & 250 & 100 & 13000 & 4100 & 4000 & 110 & 750 \\
E1 & 1.8 & 150 & 15 & 7.3 & $\leq 96$ & 0.42 & 7.0 \\
E2(LVC) & 1.0 & 150 & 3.2 & $\leq 0.44$ & $\leq 260$ & 0.21 & $\leq 0.86$ \\
E2(HVC) & 0.4 & 100 & 7.8 & $\leq 1.1$ & $\leq 1200$ & 1.0 & $\leq 1.8$ \\
W1 & 2.6 & 150 & 24 & 2.4 & $\leq 72$ & 0.15 & 3.4 \\
W2 & 1.8 & 80 & 8.7 & 2.9 & $\leq 76$ & 0.15 & 3.6 \\
Cloud & 0.3 & 26 & $30-80$ & 2 & 1.5 & 0.02 & 2 \\
\hline
\end{tabular}

Table 4. Abundances in molecular clouds. References: L1689N: SO, $\mathrm{SO}_{2}$ and $\mathrm{H}_{2} \mathrm{~S}$ from this work, $\mathrm{SiO}$ from Ceccarelli et al. (2000b) and $\mathrm{H}_{2} \mathrm{CO}$ from Ceccarelli et al. (2001); L134N: SO and $\mathrm{SO}_{2}$ abundances from Swade (1989), $\mathrm{H}_{2} \mathrm{~S}$ from Minh et al. (1989), $\mathrm{SiO}$ from Ziurys et al. (1989), $\mathrm{H}_{2} \mathrm{CO}$ from Ohishi et al. (1992); TCM-1: SO abundances from Hirahara et al. (1995) and Pratap et al. (1997), $\mathrm{SO}_{2}$ from Irvine et al. (1983), $\mathrm{H}_{2} \mathrm{~S}$ from Minh et al. (1989), $\mathrm{SiO}$ from Ziurys et al. (1989), $\mathrm{H}_{2} \mathrm{CO}$ from Ohishi \& Kaifu (1998).

\begin{tabular}{c|ccccc}
\hline \hline Cloud & $x(\mathrm{SO})$ & $x\left(\mathrm{SO}_{2}\right)$ & $x\left(\mathrm{H}_{2} \mathrm{~S}\right)$ & $x(\mathrm{SiO})$ & $x\left(\mathrm{H}_{2} \mathrm{CO}\right)$ \\
& $\left(10^{-9}\right)$ & $\left(10^{-9}\right)$ & $\left(10^{-9}\right)$ & $\left(10^{-12}\right)$ & $\left(10^{-8}\right)$ \\
\hline L1689N & $6-16$ & 0.4 & 0.3 & 4 & 0.04 \\
L134N & $0.6-10$ & $0.3-2.5$ & 3 & $\leq 3.6$ & 2 \\
TMC-1 & $0.3-4$ & $2-6$ & 0.7 & $\leq 2.4$ & 7 \\
\hline
\end{tabular}

respectively ${ }^{2}$. The column densities derived for all species are reported in Table 3 and the abundances in Table 4.

\subsection{Shocked regions}

We have used the gas temperature and density derived by CCLCL01 in E1, E2(LVC), E2(HVC), W1, $\mathrm{W}^{3}$, i.e. in the shocked regions, by comparing the $\mathrm{SiO}$ observed lines ( $J$ from 2 to 5 ) with the relevant LVG model. The uncertainty in the derived densities and temperatures is around a factor two. We did not try to derive independently the gas temperature and density for each molecule, because of the lack of

\footnotetext{
2 Although no $\mathrm{SiO}$ emission was detected towards the cloud position, Ceccarelli et al. (2000b) modeled the observed $\mathrm{SiO}$ (from $J_{\text {up }}=1$ to 8 ) emission towards IRAS 16293 , taking into account the envelope physical structure, and found that the $\mathrm{SiO}$ abundance is $4.0 \times 10^{-12}$ in the outer envelope. In analogy with other molecules for which the abundances found in the outer envelope are equal to the (measured) abundances in the surrounding molecular clouds, we assumed $4.0 \times$ $10^{-12}$ for the $\mathrm{SiO}$ abundance in L1689N. In a similar way, although weak formaldehyde emission is detected at the cloud position, we used the abundance found in the IRAS 16293 outer envelope for the cloud too.

3 Since no $\mathrm{SiO}$ emission is detected in HE2, the density and temperature cannot be constrained in this position.
}

enough usable transitions. In fact, of the two SO transitions, the lowest lying transition is dominated by the cloud emission so it cannot be used to probe the shocked gas, and only one transition of $\mathrm{SO}_{2}, \mathrm{H}_{2} \mathrm{~S}$ and $\mathrm{H}_{2} \mathrm{CO}$ respectively has been detected. We therefore derived the column densities of those last four species assuming the same density and temperature derived from the $\mathrm{SiO}$ observations. This is an approximation that does not take into account the possible structure of the shocked gas, but the results are indeed not much affected by this assumption. In principle each molecular species may originate in slightly different physical conditions and hence the computed column density may be consequently mis-evaluated. In practice, though, the error associated with this approximation is lower than about a factor two. For example, Lis et al. (2002), using $\mathrm{H}_{2} \mathrm{CO}$ transitions, found different values for the temperature $(45 \mathrm{~K})$ and density $\left(2 \times 10^{6} \mathrm{~cm}^{-3}\right)$ in E1. Even taking those values, the derived column density of the four molecules listed in Table 3 wouldn't change by more than a factor two with respect to those quoted in the table. Finally, we also assumed that all the used lines are optically thin (e.g. Blake et al. 1994). The column densities, averaged on the relevant beam, derived for all species in each of the four shocked sites are reported in Table 3. Unfortunately, since we don't have observations of the $\mathrm{H}_{2} \mathrm{~S}$ lowest transitions in the shocks, the column density of this molecule is very poorly constrained.

\subsection{IRAS 16293}

As mentioned in the Introduction, the envelope of IRAS 16293 is formed by (at least) two components: an inner core characterized by a high temperature and an outer cold envelope with a temperature close to the cloud temperature. The hot core is a small region $\left(\sim 2^{\prime \prime}\right.$ in diameter) whose temperature is about $100 \mathrm{~K}$ and the density $2.5 \times 10^{7} \mathrm{~cm}^{-3}$ (Ceccarelli et al. 2000a; Schöier et al. 2002). At that temperature the grain mantles evaporate injecting into the gas phase their constituents (Ceccarelli et al. 2000a,b). The outer envelope is more extended $\left(\sim 20^{\prime \prime}\right)$ and colder, with a temperature of $30 \mathrm{~K}$ (a little larger than the $\mathrm{L} 1689 \mathrm{~N}$ temperature) and a density of $2.5 \times 10^{5} \mathrm{~cm}^{-3}$ (Ceccarelli et al. 2000a; Schöier et al. 2002). 
The molecular abundances in the outer envelope are similar to those in the molecular cloud, with the possible exception of the deuterated molecules. On the contrary, in the inner core, several molecules, those believed to be released from the grain mantles or formed from those evaporated molecules, undergo a jump in their abundances. In the following we focus on the abundance of the S-bearing molecules in the inner hot core. Blake et al. (1994), using multifrequency observations, constructed the rotational diagrams of $\mathrm{SO}$ and $\mathrm{SO}_{2}$ and derived rotational temperatures relatively large $(\sim 80 \mathrm{~K})$. It is therefore very likely that the bulk of the emission for both molecules originates in the inner core. Furthermore, all linewidths are larger than $3 \mathrm{~km} \mathrm{~s}^{-1}$ and increase with the upper level energy of the transition, once again arguing for an inner core origin. Pursuing this hypothesis we computed the column densities using these rotational diagrams, corrected for the beam dilution, reported in Table 3. In estimating the $\mathrm{H}_{2} \mathrm{~S}$ column density we assumed that the $2_{2,0} \rightarrow 2_{1,1}$ line emission originates entirely in the hot core, as it is suggested by its observed relatively large linewidth. Using then the estimates of the $\mathrm{H}_{2}$ column density in Ceccarelli et al. (2000a) $\left(7.5 \times 10^{22} \mathrm{~cm}^{-2}\right)$ we derive the abundances reported in Table 5. Note that, provided that we use our volume density in the Schöier et al. (2002) model, our derived abundance compare extremely well with their abundances (which, as a result, is multiplied by a factor five), supporting the validity of our method. Actually Schöier et al. found abundances a factor 5 lower than ours, because they used a factor five larger density than us in the inner core. The two density estimates differ because of the different diagnostics used to derive them: Schöier et al. used the continuum spectral energy distribution, whereas Ceccarelli et al. used the water line spectrum. When CO lines are used instead, Schöier et al. found the same density in the inner region (Schöier private communication). We use therefore the estimate by Ceccarelli et al. (2000a), but keep in mind that we may be overestimating the abundances by a factor 5 . Finally, the $\mathrm{SiO}$ and $\mathrm{H}_{2} \mathrm{CO}$ column densities are taken from the Ceccarelli et al. (2000a,b, 2001) modeling.

\section{Discussion}

The abundance ratios between the observed species are reported in Tables 4-6. They show variations up to two orders of magnitude. In the following we analyze in detail the variations associated with the cloud, the shocked regions and the protostar IRAS 16293 respectively.

\subsection{The cloud}

Adopting the $\mathrm{H}_{2}$ column density derived by Caux et al. (1999) by means of atomic oxygen observations in L1689N, $5 \times$ $10^{22} \mathrm{~cm}^{-2}$, and using the column densities derived in the previous section (Table 3 ) we obtain the abundances reported in Table 4. In the table we also report, for comparison, the abundances measured in L134N and TMC-1, two among the best studied molecular clouds. With the possible exception of formaldehyde, which seems to be underabundant, L1689N has abundances typical of other cold molecular clouds and it is therefore interesting to study how those abundances change in the region either because of the presence of shocks or the presence of a protostar (IRAS 16293).

\subsection{Shocked regions}

In the shocked regions, with the exception of E2, it is practically impossible to derive the absolute abundances of the observed molecules, because of the difficulty to derive reliable estimates of the $\mathrm{H}_{2}$ column densities. In fact, usually the $\mathrm{H}_{2}$ column densities are estimated from the $\mathrm{CO}$ millimeter observations converting the measured $\mathrm{CO}$ column densities into $\mathrm{H}_{2}$ column densities. The method relies on the capacity to disentangle the contribution of the cloud from the shocked gas in the $\mathrm{CO}$ emission. However, this is feasible when the shocked gas emits at relatively large velocities, i.e. when the two contributions can be separated based on their spectral properties (see for example Bachiller \& Perez Gutierrez 1997). In the specific case of the outflows in L1689N, the projected velocity of the shocks is too small and the cloud and shocked gas cannot be disentangled, except in the E2 position, where a high velocity component is present. In order therefore to estimate the $\mathrm{H}_{2}$ column density in the shocked regions we would need high spatial resolution observations, because single dish CO measurement are totally dominated by the cloud emission. For this practical reason, we computed only abundances ratios, as summarized in Table 6. Note that the quoted ratios have been obtained dividing column densities averaged on different beam sizes ( $18^{\prime \prime}$ for $\mathrm{SO}_{2}$ observations and $\sim 25^{\prime \prime}$ for the others), so that the $\mathrm{SO}_{2}$ ratios may be off by a factor two (in the shocked regions). Figure 5 shows a graphic representation of the derived abundances ratios in the shocked regions and in the cloud.

The first result to note is that, with the exception of the $\mathrm{SO}_{2} / \mathrm{H}_{2} \mathrm{CO}$ ratio, all other ratios in the shocked regions differ by more than a factor two when compared to the ratios in the cloud. In particular, the largest variations, by up a factor 100 , are shown by the ratios with the $\mathrm{SiO}$, confirming that $\mathrm{SiO}$ is the best molecule to trace shocks (Caselli et al. 1997; Schilke et al. 1997; Garay et al. 2002).

A second robust result is that $\mathrm{SO}_{2}$ is enhanced with respect to SO in the E1, W1 and W2 shocks, when compared to the cloud (by up a factor 10). This also confirms a rather general theoretical prediction that $\mathrm{SO}_{2}$ is overproduced with respect to $\mathrm{SO}$ in the shocked gas, because of endothermic reactions that lock most of the gaseous sulphur in $\mathrm{SO}_{2}$ on timescales relatively small $\sim 10^{3}$ yr (e.g. Pineau Des Forêts et al. 1993). Remarkably, $\mathrm{SO}_{2} / \mathrm{SO}$ is lowest in the strongest $\mathrm{SiO}$ shock of the region, E2. CCLCL01 argued that E2 is an older shock based on $\mathrm{H}_{2} \mathrm{CO}$ and $\mathrm{SiO}$ observations compared with models predictions and this would agree with the low $\mathrm{SO}_{2} / \mathrm{SO}$ ratio, as $\mathrm{SO}_{2}$ is expected to be converted into atomic sulphur at late stages $\geq 3 \times 10^{4} \mathrm{yr}$ (e.g. Hatchell et al. 1998).

The third result, is the constant $\mathrm{SO}_{2} / \mathrm{H}_{2} \mathrm{CO}$ ratio, within a factor 2, in the shocked regions E1, W1 and $\mathrm{W} 2$, and in the cloud. Why $\mathrm{SO}_{2} / \mathrm{H}_{2} \mathrm{CO}$ has the same value in these shocked regions and in the cloud seems a remarkable coincidence for which we do not have an explanation. On the contrary, the constant $\mathrm{SO}_{2} / \mathrm{H}_{2} \mathrm{CO}$ ratio in these shocked regions may be due 
Table 5. Abundances of $\mathrm{SO}, \mathrm{SO}_{2}, \mathrm{H}_{2} \mathrm{~S}, \mathrm{SiO}$ and $\mathrm{H}_{2} \mathrm{CO}$ with respect to $\mathrm{H}_{2}$ in the hot core of IRAS 16293 . The abundances of $\mathrm{SO}$, $\mathrm{SO}_{2}$ and $\mathrm{H}_{2} \mathrm{~S}$ are estimated by the present work observations, assuming a $\mathrm{H}_{2}$ column density of $7.5 \times 10^{22} \mathrm{~cm}^{-2}$ (Ceccarelli et al. 2000a). The SiO and $\mathrm{H}_{2} \mathrm{CO}$ abundances are taken from Ceccarelli et al. (2000a,b). References of the other sources: L1157-mm (IRAS 20386+6751): Bachiller \& Perez Gutierrez (1997); Orion-KL: SO, $\mathrm{SO}_{2}, \mathrm{SiO}$ and $\mathrm{H}_{2} \mathrm{CO}$ from Sutton et al. (1995), $\mathrm{H}_{2} \mathrm{~S}$ from Minh et al. (1990); G10.47, G29.96, G75.78, G9.62, G12.21, G31.41 and G34.26: Hatchell et al. (1998); BF-Class 0: Buckle \& Fuller (2003).

\begin{tabular}{lccccc}
\hline \hline & $\mathrm{SO} / \mathrm{H}_{2}$ & $\mathrm{SO}_{2} / \mathrm{H}_{2}$ & $\mathrm{H}_{2} \mathrm{~S} / \mathrm{H}_{2}$ & $\mathrm{SiO} / \mathrm{H}_{2}$ & $\mathrm{H}_{2} \mathrm{CO} / \mathrm{H}_{2}$ \\
\hline IRAS 16293 & $1.7 \times 10^{-6}$ & $5.4 \times 10^{-7}$ & $5.3 \times 10^{-7}$ & $1.5 \times 10^{-8}$ & $1.0 \times 10^{-7}$ \\
L1157-mm & $5.0 \times 10^{-9}$ & $3.0 \times 10^{-8}$ & $1.1 \times 10^{-8}$ & - & $4-6 \times 10^{-9}$ \\
Orion-KL & $1.5 \times 10^{-7}$ & $9.4 \times 10^{-8}$ & $5.0 \times 10^{-6}$ & $6.0 \times 10^{-9}$ & $7.0 \times 10^{-9}$ \\
G10.47 & $3.0 \times 10^{-9}$ & $1.8 \times 10^{-8}$ & $\geq 4.0 \times 10^{-9}$ & - & - \\
G29.96 & $\geq 5.0 \times 10^{-9}$ & $2.0 \times 10^{-8}$ & $\geq 1.5 \times 10^{-8}$ & - & - \\
G75.78 & $3.0 \times 10^{-9}$ & $\geq 2.0 \times 10^{-9}$ & $\geq 1.0 \times 10^{-9}$ & - & - \\
G9.62 & $\geq 4.0 \times 10^{-9}$ & $1.0 \times 10^{-8}$ & $\geq 8.0 \times 10^{-9}$ & - & - \\
G12.21 & $\geq 8.0 \times 10^{-10}$ & $\geq 5.0 \times 10^{-10}$ & & - & - \\
G31.41 & $4.0 \times 10^{-9}$ & $1.2 \times 10^{-8}$ & $\geq 3.0 \times 10^{-9}$ & - & - \\
G34.26 & $6.0 \times 10^{-9}$ & $1.5 \times 10^{-8}$ & $\geq 2.0 \times 10^{-9}$ & - & - \\
BF-Class 0 & $3.0 \times 10^{-9}$ & $4.0 \times 10^{-10}$ & $2.0 \times 10^{-9}$ & - & - \\
\hline
\end{tabular}

Table 6. Abundance ratios in the shocked regions and in the cloud, as derived from the column densities of Table 3. References for other outflows: L1157-B1 and L1157-B2: Bachiller \& Perez Gutierrez (1997); CB3: Codella \& Bachiller (1999); L1448, B1 and CepA: Martin-Pintado et al. (1992); NGC 2071: Chernin \& Masson (1993).

\begin{tabular}{c|ccccccc}
\hline \hline Region & $\mathrm{SO} / \mathrm{H}_{2} \mathrm{CO}$ & $\mathrm{SO}_{2} / \mathrm{SO}$ & $\mathrm{SO}_{2} / \mathrm{H}_{2} \mathrm{CO}$ & $\mathrm{H}_{2} \mathrm{~S} / \mathrm{SO}$ & $\mathrm{SiO} / \mathrm{SO}$ & $\mathrm{SiO} / \mathrm{SO}_{2}$ & $\mathrm{SiO} / \mathrm{H}_{2} \mathrm{CO}$ \\
Cloud & 28 & $\sim 0.04$ & 1 & $\sim 0.03$ & $\sim 4 \times 10^{-4}$ & 0.01 & 0.01 \\
\hline E1 & 2 & 0.5 & 1.1 & $\leq 6$ & 0.03 & 0.06 & 0.06 \\
E2(LVC) & $\geq 4$ & $\leq 0.1$ & - & $\leq 80$ & 0.06 & $\geq 0.5$ & $\geq 0.2$ \\
E2(HVC) & $\geq 5$ & $\leq 0.1$ & - & $\leq 150$ & 0.1 & $\geq 0.9$ & $\geq 0.5$ \\
W1 & 7 & 0.1 & 0.7 & $\leq 3$ & $6 \times 10^{-3}$ & 0.06 & 0.04 \\
W2 & 2.5 & 0.3 & 0.8 & $\leq 9$ & 0.02 & 0.05 & 0.04 \\
\hline L1157-B1 & $0.6-1$ & $0.6-1$ & $0.4-1$ & $0.8-1.3$ & $0.2-0.3$ & 0.3 & $0.1-0.3$ \\
L1157-B2 & 1 & $1-3$ & $1-3$ & $0.7-2$ & $0.1-0.4$ & 0.1 & $0.1-0.4$ \\
CB3 & - & $\sim 1$ & - & $\leq 1$ & $\ll 1$ & $\ll 1$ & - \\
L1448 & - & - & - & - & 0.3 & - & - \\
B1 & - & - & - & - & 0.1 & - & - \\
CepA & - & - & - & - & 0.08 & - & - \\
NGC 2071 & - & - & - & - & $0.01-0.05$ & - & - \\
\hline
\end{tabular}

to the timescales involved in the formation and destruction of the two molecules, even though the mechanisms of $\mathrm{SO}_{2}$ and $\mathrm{H}_{2} \mathrm{CO}$ formation in shocks are expected to be different. On the one hand, formaldehyde is thought to be formed onto the grain mantles, released into the gas phase because of the mantle sputtering in the shock, and finally converted into more complex molecules by gas phase reactions on timescales larger than $\sim 3 \times$ $10^{4} \mathrm{yr}$ (Charnley et al. 1992). $\mathrm{SO}_{2}$ follows a totally different route: it has been proposed that sulphur is released into the gas phase mainly as $\mathrm{H}_{2} \mathrm{~S}$, following also mantle sputtering, and then $\mathrm{H}_{2} \mathrm{~S}$ is transformed into $\mathrm{SO}$ and $\mathrm{SO}_{2}$ via neutral-neutral reactions on timescales of $\sim 10^{3} \mathrm{yr}$ (e.g. Charnley 1997). Later on $\sim 3 \times 10^{4} \mathrm{yr}$, gaseous sulphur is expected to be in atomic sulphur. Therefore, the constant $\mathrm{SO}_{2} / \mathrm{H}_{2} \mathrm{CO}$ ratio in E1, W1 and W2 may tell us that these three shocks have similar ages, around $10^{4} \mathrm{yr}$ if models are correct, whereas E2 is a much older shock, $\geq 3 \times 10^{4} \mathrm{yr}$, and $\mathrm{SO}_{2}$ and $\mathrm{H}_{2} \mathrm{CO}$ both disappear because transformed into $\mathrm{S}$ and complex O-bearing molecules respectively.

The overall emerging picture is that the combination of the variations in the abundance of different molecules can in first instance give an estimate of the age of the shock. Table 7 summarizes the situation. The times noted in the Table 7 are really modeled dependent and are meant to represent a sequence. 


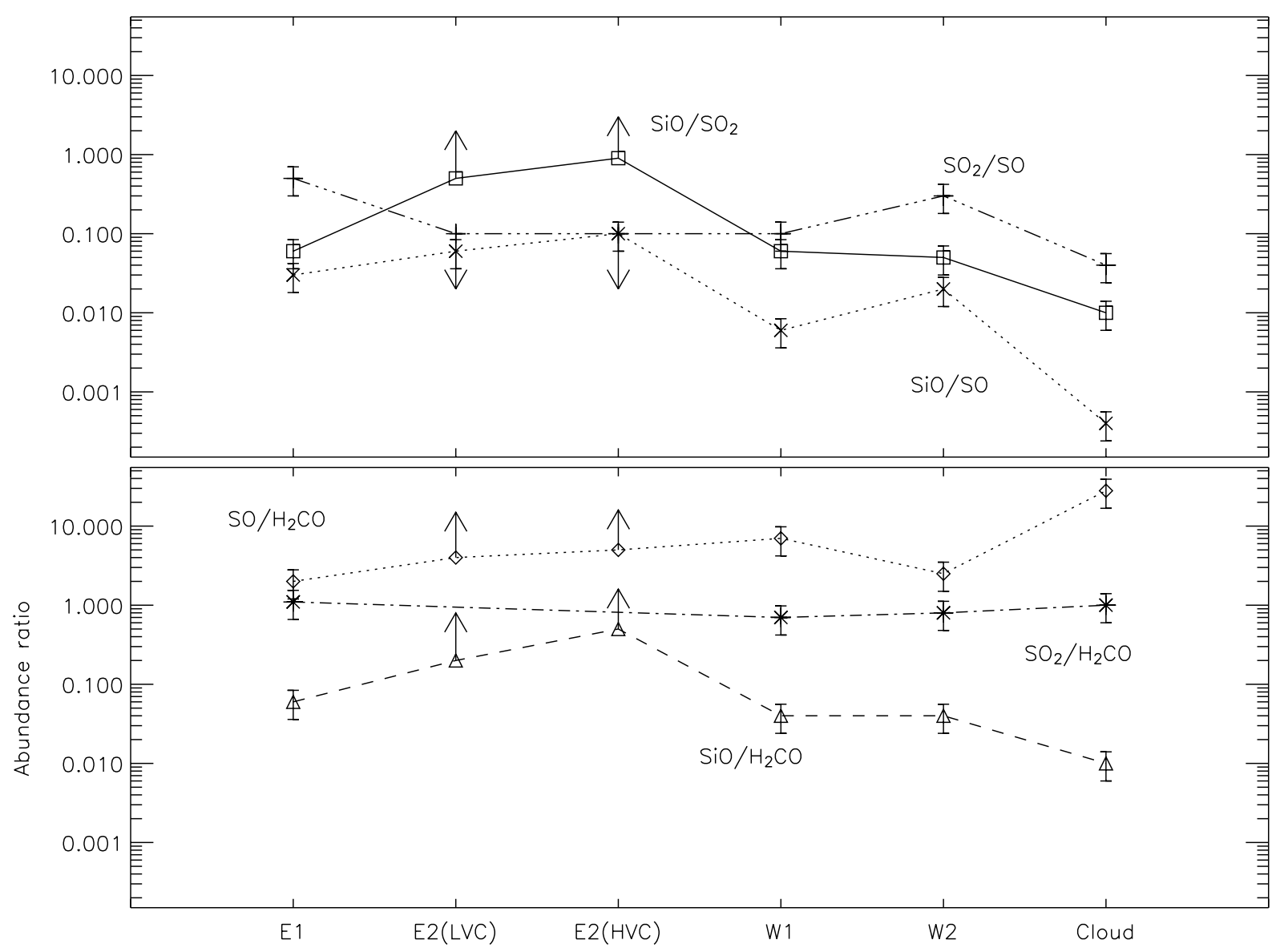

Fig. 5. The abundance ratios observed in the different shocked regions and in the molecular cloud and presented in $\mathrm{Table} 6: \mathrm{SO} / \mathrm{H}_{2} \mathrm{CO}$, $\mathrm{SO}_{2} / \mathrm{H}_{2} \mathrm{CO}$ and $\mathrm{SiO} / \mathrm{H}_{2} \mathrm{CO}$ on the lower panel and $\mathrm{SO}_{2} / \mathrm{SO}, \mathrm{SiO} / \mathrm{SO}_{2}$ and $\mathrm{SiO} / \mathrm{SO}$ on the upper panel. The arrows represent upper and lower limits of the $\mathrm{SO}_{2} / \mathrm{SO}, \mathrm{SiO} / \mathrm{SO}_{2}, \mathrm{SO} / \mathrm{H}_{2} \mathrm{CO}$ and $\mathrm{SiO} / \mathrm{H}_{2} \mathrm{CO}$ ratios respectively.

Table 7. Proposed schema of the formation and destruction of SO, $\mathrm{SO}_{2}, \mathrm{H}_{2} \mathrm{~S}, \mathrm{SiO}$ and $\mathrm{H}_{2} \mathrm{CO}$ as function of time. The times are indicative and are meant to represent a sequence.

\begin{tabular}{ccc}
\hline \hline Time $(\mathrm{yr})$ & Formation & Destruction \\
\hline $0^{a}$ & $\mathrm{H}_{2} \mathrm{~S}, \mathrm{H}_{2} \mathrm{CO}, \mathrm{SiH}_{2}, \mathrm{SiH}_{4}$ & \\
$10^{3}$ & $\mathrm{SO}, \mathrm{SO}_{2}, \mathrm{H}_{2} \mathrm{~S}, \mathrm{SiO}$ & \\
$3 \times 10^{4}$ & & $\mathrm{SO}_{2}, \mathrm{H}_{2} \mathrm{CO}$ \\
$10^{5}$ & & $\mathrm{SiO}$ \\
\hline
\end{tabular}

${ }^{a}$ Injection in the gas phase of the molecules sputtered from the grain mantles.

The simultaneous presence of $\mathrm{SO}, \mathrm{SO}_{2}, \mathrm{H}_{2} \mathrm{~S}, \mathrm{H}_{2} \mathrm{CO}$, and $\mathrm{SiO}$ would mark relatively young shocks $\left(\leq 10^{4} \mathrm{yr}\right)$, whereas the only presence of $\mathrm{SiO}$ emission would point towards relatively older shocks $\left(\geq 3 \times 10^{4} \mathrm{yr}\right)$.

Finally, comparison with other molecular outflows can only be very approximate, but still somewhat illustrative (Table 6). The only molecular outflow at our knowledge in which SO, $\mathrm{SO}_{2}, \mathrm{SiO}$ and $\mathrm{H}_{2} \mathrm{CO}$ abundances have been measured is the L1157 outflow (Bachiller \& Perez Gutierrez 1997). Looking at Table 6, L1157 B1 and B2 are rather similar to E2 with respect to the ratios involving $\mathrm{SiO}$, i.e. they are relatively enriched in $\mathrm{SiO}$ with respect to the other shocks of L1689N and the molecular cloud. The likely interpretation is that E2, B1 and B2 are all relatively strong shocks. Yet, contrary to what happens in E2, $\mathrm{H}_{2} \mathrm{CO}$, SO and $\mathrm{SO}_{2}$ are detected in $\mathrm{L} 1157 \mathrm{~B} 1$ and $\mathrm{B} 2$, which would point towards relatively young shocks (Table 7). Note though that SO seems to be underabundant with respect to $\mathrm{H}_{2} \mathrm{CO}$ and $\mathrm{SiO}$ in $\mathrm{B} 1$ and $\mathrm{B} 2$ when compared not only to $\mathrm{E} 2$, but also to E1, W1 and W2. At the same time, $\mathrm{SO}_{2}$ is overabundant. Whether this is because of the stronger shock in $\mathrm{B} 1$ and $\mathrm{B} 2$ that converts $\mathrm{SO}$ into $\mathrm{SO}_{2}$ more quickly, or because the shocks in L1157 have a different age of E1, W1 and W2, is impossible to say at this stage. A larger statistics on a larger number of outflow system is necessary to better understand this point.

\subsection{IRAS 16293}

Table 5 quotes the absolute abundances of $\mathrm{SO}, \mathrm{SO}_{2}, \mathrm{H}_{2} \mathrm{~S}$, $\mathrm{H}_{2} \mathrm{CO}$ and $\mathrm{SiO}$ in the hot core of IRAS 16293 and in the molecular cloud. As evident from the table, the sulphuretted molecule abundances exhibit a strong enhancement with respect to the molecular cloud abundances. The abundance of SO increases by a factor $200, \mathrm{SO}_{2}$ by a factor 1300 and $\mathrm{H}_{2} \mathrm{~S}$ by a factor 1700 (see also Schöier et al. 2002). 


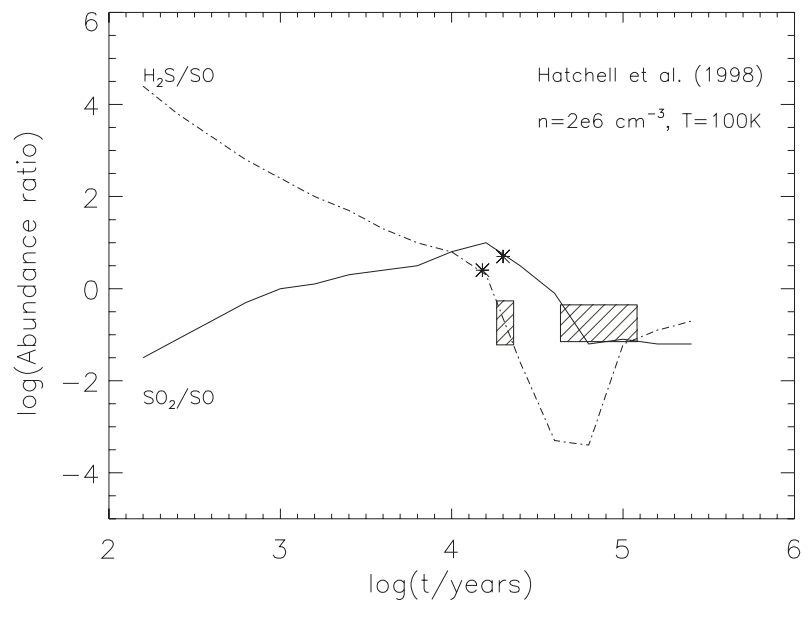

Fig. 6. Theoretical predictions of the evolution of $\mathrm{SO}_{2} / \mathrm{SO}$ and $\mathrm{H}_{2} \mathrm{~S} / \mathrm{SO}$ ratios as a function of time. The curves are adapted from the Hatchell et al. (1998) model, that assumes a gas temperature of $100 \mathrm{~K}$ and a density of $2 \times 10^{6} \mathrm{~cm}^{-3}$. The grey squares represent the portion of the modelling curve in agreement with observations of the hot core of IRAS 16293 (this work). Analogously, the stars mark the predicted values of the ratios in agreement with what observed towards L1157-mm (Bachiller \& Perez Gutierrez 1997).

As we mentioned in the Introduction, the sulphur species are expected to be useful to evaluate the age of hot cores. We could not resist the temptation to compare the measured abundance ratios with published theoretical predictions. In Fig. 6 we compare the $\mathrm{H}_{2} \mathrm{~S} / \mathrm{SO}$ and $\mathrm{SO}_{2} / \mathrm{SO}$ ratios obtained in the IRAS 16293 hot core with the theoretical values predicted by chemical models. The observed $\mathrm{H}_{2} \mathrm{~S} / \mathrm{SO}$ and $\mathrm{SO}_{2} / \mathrm{SO}$ ratios are consistent with the theoretical predictions by Hatchell et al. (1998), but differ substantially with those by Charnley (1997). Both models use the sulphur chemistry described in Charnley (1997) and assume that the bulk of sulphur is stored in iced $\mathrm{H}_{2} \mathrm{~S}$ onto the grain mantles. When the dust temperature exceeds $100 \mathrm{~K}$, the grain mantles evaporate releasing the $\mathrm{H}_{2} \mathrm{~S}$ into the gas phase, which is slowly converted into $\mathrm{SO}$ and subsequently into $\mathrm{SO}_{2}$ by neutral-neutral reactions. The only difference between the two models is that Hatchell et al. use an updated rate for the reaction of $\mathrm{H}_{2} \mathrm{~S}$ with atomic hydrogen. This difference substantially changes the evolution of the abundances of $\mathrm{H}_{2} \mathrm{~S}, \mathrm{SO}$ and $\mathrm{SO}_{2}$ at times larger than about $10^{4} \mathrm{yr}$. Our observations support the Hatchell et al. (1998) model, as shown in Fig. 6. The agreement between observations and model predictions for an age of $\sim 3 \times 10^{4} \mathrm{yr}$ is remarkable, as independent estimates of the age of IRAS 16293 converge towards the same value (Ceccarelli et al. 2000a). On the same figure we also report the values observed towards L1157-mm. Based on these observations and on the model predictions, L1157-mm has about the same age of IRAS 16293. We caution that the error bars are indeed large enough to make the agreement between observed and predicted values just a coincidence. In this sense, this result may just mean an encouragement to pursue this kind of studies on a large sample of low mass protostars, where a statistical trend could be established. We have indeed started such a study on a sample of low mass protostars, whose we have derived the physical structure (i.e. density and temperature profiles) and formaldehyde abundance profiles (Maret et al. 2002).

A direct comparison between the measured abundances in the hot cores quoted in Table 5 and that of IRAS 16293 can only be done with respect to Orion-KL, as in the other sources the abundances are not corrected for the beam dilution (Hatchell et al. 1998; Buckle \& Fuller 2003). With respect to Orion-KL, the hot core of IRAS 16293 is enriched in $\mathrm{SO}, \mathrm{SO}_{2}$ and $\mathrm{H}_{2} \mathrm{CO}$, whereas it is deficient in $\mathrm{H}_{2} \mathrm{~S}$. The most plausible explanation is that the different abundances reflect a different composition of the ices (from which $\mathrm{H}_{2} \mathrm{CO}$ and $\mathrm{H}_{2} \mathrm{~S}$ evaporate), a fact now well documented by the study of deuterated molecules in high and low mass protostars (e.g. Ceccarelli 2002). However, the different gas temperature and density may also play a role. Finally, the $\mathrm{SO}_{2} / \mathrm{SO}$ and $\mathrm{H}_{2} \mathrm{~S} / \mathrm{SO}$ ratios of IRAS 16293 differ by about a factor 100 and 10 respectively with respect to the relevant ratios measured in the high mass protostars of the Hatchell et al. (1998) sample. Again, the difference may be attributed to both different evolutionary stages and different gas densities and temperatures.

Following the theoretical expectations that gaseous sulphur in the warm gas is mainly locked into $\mathrm{SO}, \mathrm{SO}_{2}$ and $\mathrm{H}_{2} \mathrm{~S}$ (e.g. Pineau Des Forêts et al. 1993; Charnley 1997), while silicon is mainly locked into $\mathrm{SiO}$ (Herbst et al. 1989), the $\left(\mathrm{SO}+\mathrm{SO}_{2}+\mathrm{H}_{2} \mathrm{~S}\right) / \mathrm{SiO}$ ratio gives a measure of the gas phase elemental sulphur over silicon abundance ratio in the IRAS 16293 hot core. This value is about 300 , to compare with the solar elemental sulphur over silicon abundance ratio of 0.5 . It is unlikely that we overestimate the overall quantity of sulphur. Conversely, it could have been underestimated because of a possible optical thickness of the sulfuretted molecular lines and/or the presence of other important sulphur-bearing molecular species not considered here. Thus, the measured $\left(\mathrm{SO}+\mathrm{SO}_{2}+\mathrm{H}_{2} \mathrm{~S}\right) / \mathrm{SiO}$ ratio suggests that there is an important deficiency of silicon in the warm gas of the IRAS 16293 hot core. The likely explanation is that silicon is mostly depleted into the non-volatile, refractory cores grains, whereas sulphur is depleted mostly onto the volatile grain mantles. This conclusion supports the thesis by Ruffle et al. (1999) that sulphur and silicon follow different routes of depletion, and specifically that sulphur is depleted at the time of mantle formation. Another possibility to explain this deficiency of silicon is that silicon is mainly in another form than $\mathrm{SiO}$ in the warm gas such as $\mathrm{Si}$, $\mathrm{Si}^{+}$or $\mathrm{SiO}_{2}$. The atomic silicon is difficult to observe, since it has its ground transition at $129 \mu \mathrm{m}$, i.e. a wavelength range obscured by the atmosphere. Observations obtained with the Long Wavelength Spectrometer on board ISO did not detect any signal larger than about $5 \times 10^{-13} \mathrm{erg} \mathrm{s}^{-1} \mathrm{~cm}^{-2}$ (Ceccarelli et al. 1998), corresponding to an upper limit for the Si column density of $6 \times 10^{17} \mathrm{~cm}^{-2}$, and an abundance lower than $8 \times 10^{-6}$, i.e. about 500 times more than the $\mathrm{SiO}$ abundance found in IRAS 16293. For $\mathrm{Si}^{+}$, it is unlikely that the UV field in the observed regions is strong enough to ionize the silicon.

Finally, the total abundance of $\mathrm{SO}, \mathrm{SO}_{2}$ and $\mathrm{H}_{2} \mathrm{~S}$ in the IRAS 16293 hot core is $2.8 \times 10^{-6}$, namely more than ten times lower than the corresponding solar abundance $3.4 \times 10^{-5}$. Even adding up the OCS, whose abundance is about that of SO (Schöier et al. 2002), the overall sulphur abundance is still 
low. Unfortunately (Schöier et al. 2002) could not estimate the CS abundance in the hot core of IRAS 16293, but CS is unlikely to be the main reservoir of sulphur. The other possible reservoir of sulphur, the atomic sulphur (Charnley 1997), is extremely difficult to observe, because the ground state transition is at $25 \mu \mathrm{m}$, i.e. in a wavelength range obscured by the atmosphere.

\section{Conclusion}

We have presented a quantitative observational study of the most important S-bearing molecules, namely $\mathrm{SO}, \mathrm{SO}_{2}$ and $\mathrm{H}_{2} \mathrm{~S}$, in the region of $\mathrm{L} 1689 \mathrm{~N}$. We derived the column density of these molecules plus $\mathrm{SiO}$ and $\mathrm{H}_{2} \mathrm{CO}$ molecules in six regions of L1689N: the cloud, the young protostar IRAS 16293, and four shocked regions.

We found that $\mathrm{SiO}$ is the molecule that shows the largest abundance variations in the shocked regions, whereas S-bearing molecules show more moderate variations. Remarkably, the region of the brightest $\mathrm{SiO}$ emission in $\mathrm{L} 1689 \mathrm{~N}$, namely E2, is undetected in $\mathrm{SO}_{2}, \mathrm{H}_{2} \mathrm{~S}$ and $\mathrm{H}_{2} \mathrm{CO}$ and only marginally detected in SO. We argued that this is possibly due to the relatively old age $\left(\geq 3 \times 10^{4} \mathrm{yr}\right)$ of this shock.

In the other weaker $\mathrm{SiO}$ shocks, $\mathrm{SO}_{2}$ is enhanced with respect to $\mathrm{SO}$, in agreement with theoretical expectations that predict the conversion of the gaseous sulphur mostly into $\mathrm{SO}_{2}$ on timescales of $\sim 10^{3} \mathrm{yr}$. In the same regions, the $\mathrm{SO}_{2} / \mathrm{H}_{2} \mathrm{CO}$ ratio is of order of unity. We argued that this may point to relatively young shocks $\left(\sim 10^{4} \mathrm{yr}\right)$, where $\mathrm{SO}_{2}$ has already formed and $\mathrm{H}_{2} \mathrm{CO}$ has not yet destroyed.

Putting together the observed combinations of the $\mathrm{SO}, \mathrm{SO}_{2}$, $\mathrm{H}_{2} \mathrm{CO}$ and $\mathrm{SiO}$ ratios, we proposed a schema in which the different molecular ratios correspond to different ages of the shocks.

Finally, we found that $\mathrm{SO}, \mathrm{SO}_{2}$ and $\mathrm{H}_{2} \mathrm{~S}$ have significant abundance jumps (200, 1300 and 1700 respectively) in the inner hot core of IRAS 16293 . We compared the measured abundances with theoretical models and discussed the derived protostar age. However, we cautioned that a more detailed study is necessary to draw reliable conclusions. The hot core of IRAS 16293 seems to be enriched in $\mathrm{SO}, \mathrm{SO}_{2}$ and $\mathrm{H}_{2} \mathrm{CO}$ with respect to Orion-KL, probably because of a different initial composition of the ices in the two sources. Comparing the $\mathrm{SO}+\mathrm{SO}_{2}+\mathrm{H}_{2} \mathrm{~S} / \mathrm{SiO}$ ratio in the hot core of IRAS 16293 , we found that silicon is largely deficient in the warm gas (by a factor $\sim 600$ ), supporting the thesis that silicon is depleted into the grain refractory cores whereas sulphur is depleted into the grain volatile mantles. Nonetheless, sulphur in the IRAS 16293 warm gas is also deficient.

Acknowledgements. We thank the IRAM and SEST staff in Pico Veleta and La Silla for their assistance with the observations, and the IRAM and ESO Program Committee for their award of observing time. We would like to thank G. Fuller, the referee, for useful comments.We are grateful to A.G.G.M. Tielens and M. Walmsley, for helpful discussions on sulphur and silicon chemistry. V. Wakelam wishes to thank F. Herpin for his help on data reduction.

\section{References}

Bachiller, R., Pérez Gutiérrez, M., Kumar, M. S. N., \& Tafalla, M. 2001, A\&A, 372, 899

Bachiller, R., \& Perez Gutierrez, M. 1997, ApJ, 487, L93

Blake, G. A., van Dishoek, E. F., Jansen, D. J., Groesbeck, T. D., \& Mundy, L. G. 1994, ApJ, 428, 680

Buckle, J. V., \& Fuller, G. A. 2003, A\&A, 399, 567

Caselli, P., Hartquist, T. W., \& Havnes, O. 1997, A\&A, 322, 296

Castets, A., Ceccarelli, C., Loinard, L., Caux, E., \& Lefloch, B. 2001, A\&A, 375, 40

Caux, E., Ceccarelli, C., Castets, A., et al. 1999, A\&A, 347, L1

Ceccarelli, C. 2002, P\&SS, 50, 1267

Ceccarelli, C., Caux, E., Wolfire, M., et al. 1998, A\&A, 331, L17

Ceccarelli, C., Castets, A., Caux, E., et al. 2000a, A\&A, 355, 1129

Ceccarelli, C., Loinard, L., Castets, A., Tielens, A. G. G. M., \& Caux, E. 2000b, A\&A, 357, L9

Ceccarelli, C., Loinard, L., Castets, A., et al. 2001, A\&A, 372, 998

Ceccarelli, C., Baluteau, J.-P., Walmsley, M., et al. 2002, A\&A, 383, 603

Charnley, S. B. 1997, ApJ, 481, 396

Charnley, S. B., Tielens, A. G. G. M., \& Millar, T. J. 1992, ApJ, 399, L71

Chernin, L., \& Masson, C. 1993, ApJ, 403, L21

Chernin, L. M., Masson, C. R., \& Fuller, G. A. 1994, ApJ, 436, 741

Chin, Y.-N., Henkel, C., Whiteoak, J. B., Langer, N., \& Churchwell, E. B. 1996, A\&A, 305, 960

Codella, C., \& Bachiller, R. 1999, A\&A, 350, 659

Fukui, Y., Sugitani, K., Takaba, H., et al. 1986, ApJ, 311, L85

Garay, G., Mardones, D., Rodríguez, L. F., Caselli, P., \& Bourke, T. L. 2002, ApJ, 567, 980

Green, S. 1991, ApJS, 76, 979

Green, S. 1994, ApJ, 434, 188

Hatchell, J., Thompson, M. A., Millar, T. J., \& MacDonald, G. H. 1998, A\&A, 338, 713

Herbst, E., Millar, T. J., Wlodek, S., \& Bohme, D. K. 1989, A\&A, 222, 205

Hirahara, Y., Masuda, A., Kawaguchi, K., et al. 1995, PASJ, 47, 845

Hirano, N., Mikami, H., Umemoto, T., Yamamoto, S., \& Taniguchi, Y. 2001, ApJ, 547, 899

Hollenbach, D. J. 1998, in Herbig-Haro flows and the birth of low mass stars, ed. B. Reipurth \& C. Bertout, IAU Symp., 182, 181

Irvine, W. M., Good, J. C., \& Schloerb, F. P. 1983, A\&A, 127, L10

Keane, J. V., Boonman, A. M. S., Tielens, A. G. G. M., \& van Dishoeck, E. F. 2001, A\&A, 376, L5

Knude, J., \& Hog, E. 1998, A\&A, 338, 897

Lis, D. C., Gerin, M., Phillips, T. G., \& Motte, F. 2002, ApJ, 569, 322

Loinard, L., Castets, A., Ceccarelli, C., Caux, E., \& Tielens, A. G. G. M. 2001, ApJ, 552, L163

Looney, L. W., Mundy, L. G., \& Welch, W. J. 2000, ApJ, 529, 477

Lucas, R., \& Liszt, H. 1998, A\&A, 337, 246

Maret, S., Ceccarelli, C., Caux, E., Tielens, A. G. G. M., \& Castets, A. 2002, A\&A, 395, 573

Martin-Pintado, J., Bachiller, R., \& Fuente, A. 1992, A\&A, 254, 315

Millar, T. J., \& Herbst, E. 1990, A\&A, 231, 466

Minh, Y. C., Irvine, W. M., \& Ziurys, L. M. 1989, ApJ, 345, L63

Minh, Y. C., Irvine, W. M., McGonagle, D., \& Ziurys, L. M. 1990, ApJ, 360, 136

Minh, Y. C., Ziurys, L. M., Irvine, W. M., \& McGonagle, D. 1991, ApJ, 366, 192

Mizuno, A., Fukui, Y., Iwata, T., Nozawa, S., \& Takano, T. 1990, ApJ, 356,184

Narayanan, G., Walker, C. K., \& Buckley, H. D. 1998, ApJ, 496, 292 
Ohishi, M., Irvine, W. M., \& Kaifu, N. 1992, in Astrochemistry of Cosmic Phenomena, IAU Symp., 150, 171

Ohishi, M., \& Kaifu, N. 1998, in Chemistry and Physics of Molecules and Grains in Space, Faraday Discussions, 109, 205

Oppenheimer, M., \& Dalgarno, A. 1974, ApJ, 187, 231

Palma, A. 1987, ApJS, 64, 565

Pickett, H. M., Poynter, R. L., Cohen, E. A., et al. 1998, J. Quant. Spectrosc. Radiat. Transfer, 60, 883

Pineau Des Forêts, G., Roueff, E., Schilke, P., \& Flower, D. R. 1993, MNRAS, 262, 915

Prasad, S. S., \& Huntress, W. T. 1982, ApJ 260, 590

Pratap, P., Dickens, J. E., Snell, R. L., et al. 1997, ApJ, 486, 862

Ruffle, D. P., Hartquist, T. W., Caselli, P., \& Williams, D. A. 1999, MNRAS, 306, 691

Schilke, P., Walmsley, C. M., Pineau Des Forets, G., \& Flower, D. R. 1997, A\&A, 321, 293
Schöier, F. L., Jorgensen, J. K., van Dishoeck, E. F., \& Blake, G. A. 2002, A\&A, 390, 1001

Sutton, E. C., Peng, R., Danchi, W. C., et al. 1995, ApJS, 97, 455

Swade, D. A. 1989, ApJ, 345, 828

Turner, B. E. 1996, ApJ, 468, 694

Turner, B. E., Chan, K., Green, S., \& Lubowich, D. A. 1992, ApJ, 399, 114

van Dishoeck, E. F., \& Blake, G. A. 1998, ARA\&A, 36, 317

Walker, C. K., Lada, C. J., Young, E. T., Maloney, P. R., \& Wilking, B. A. 1986, ApJ, 309, L47

Wilson, T. L., \& Rood, R. 1994, ARA\&A, 32, 191

Wootten, A., \& Loren, R. B. 1987, ApJ, 317, 220

Zhou, S. 1995, ApJ, 442, 685

Ziurys, L. M., Friberg, P., \& Irvine, W. M. 1989, ApJ, 343, 201 